\title{
Phenotypic and Molecular Diversity of Cochliobolus sativus Populations from Wheat
}

Suraj Gurung, Department of Plant Pathology, University of California, Davis, c/o U.S. Agricultural Research Station, Salinas, CA 93905; Baidya N. Mahto, Division of Plant Pathology, Nepal Agricultural Research Council, Khumaltar, Lalitpur, Nepal; Sanjaya Gyawali, Agriculture and Agri-Food Canada, Saskatoon Research Centre, 107 Science Place, Saskatoon, Saskatchewan, S7N 0X2, Canada; and Tika B. Adhikari, Center for Integrated Pest Management and Department of Plant Pathology, North Carolina State University, 840 Main Campus Drive, Partners II Suite 1400, Centennial Campus, Raleigh 27606

\begin{abstract}
Gurung, S., Mahto, B. N., Gyawali, S., and Adhikari, T. B. 2013. Phenotypic and molecular diversity of Cochliobolus sativus populations from wheat. Plant Dis. 97:62-73.

Spot blotch, caused by Cochliobolus sativus, is a devastating foliar disease of wheat in Nepal and in the Northern Great Plains of the United States. However, limited information on variation in virulence and genetic structure of $C$. sativus from wheat is available. In this study, pathogenic variation of 96 isolates of $C$. sativus from the Hill and Plain areas in Nepal $(n=48)$ and in the Central and Northern areas in North Dakota $(n=48)$ were evaluated on 12 differential wheat lines. DNA polymorphisms in all isolates were analyzed using eight selected amplified fragment length polymorphism primer combinations. Phenotypic data analysis showed the isolates varied greatly and were classified into 47 pathotypes. Cluster analysis indicated the isolates fell into

three distinct groups with low, intermediate, and high virulence. Population genetic analysis revealed significant linkage disequilibrium $\left(\bar{r}_{d}\right.$ $=0.066$ to 0.292 ), indicating that sexual reproduction plays little or no role in evolution and disease epidemiology in wheat fields. Furthermore, the corrected standardized fixation index $\left(G_{\text {ST }}^{\prime \prime}=0.05\right.$ and 0.02$)$ showed no evidence of genetic differentiation in $C$. sativus populations. Collectively, these results confirmed high pathogenic and molecular diversity in the C. sativus populations collected from wheat foliar infections and will be useful to assist in developing resistant cultivars to manage this disease.
\end{abstract}

Spot blotch, caused by Cochliobolus sativus (S. Ito \& Kurib.) Drechsler ex Dastur (anamorph Bipolaris sorokiniana (Sacc.) Shoemaker), is an important disease of wheat and barley worldwide $(23,43,56)$. Duveiller et al. (12) reported that seed was the primary source of inoculum, for wheat in South Asia. However, the airborne conidia occurring from soil debris, crop residues, and wild grasses can also act as primary inoculum sources (32). Spot blotch is less problematic under dry and cool weather conditions, whereas warm humid conditions increase disease severity (12). The teleomorph of this fungus has been reported from Zambia under natural conditions (36). However, in general, the sexual stage is uncommon and contributes little to genetic variation in this fungus (56).

Knowledge of the pathogenic and genetic structure of the pathogen populations has implications for formulating disease management strategies and developing effective resistance breeding programs (37). The virulence of $C$. sativus populations from barley has been analyzed in Australia, Canada, and the United States $(17,18,23,48,56)$. Pathotype 2 was reported to be common in North Dakota and had lower allelic diversity compared with pathotypes 0 and 1 (56). Recently, Knight et al. (23) identified 11 pathotypes of C. sativus from leaves and roots of both barley and wheat from Australia. C. sativus in wheat has been reported from North America, South America, South and Central Asia, Australia, Europe, and Africa $(5,26,42,52)$. In particular, this pathogen is regarded as a major biotic stress, limiting production of wheat in the Northern

Corresponding author: T. B. Adhikari,

E-mail: tika_adhikari@ncsu.edu

* The $e$-Xtra logo stands for "electronic extra" and indicates that a supplementary table is included in the online edition.

Accepted for publication 22 July 2012.

http://dx.doi.org/10.1094/PDIS-01-12-0092-RE

(C) 2013 The American Phytopathological Society
Great Plains (53) and barley in the Canadian Prairie Provinces in North America $(17,18)$. In addition, $C$. sativus has been reported to be one of the most destructive pathogens in wheat in warm areas, particularly in the intensive rice-wheat cropping system (12), and has significant impact on wheat production in South Asia $(22,42,44)$.

Analysis of virulence and population genetic structure of $C$. sativus can assist plant pathologists and breeders in deploying resistance genes in wheat breeding programs. However, little information on pathogenic variation and genetic structure of $C$. sativus from wheat in Nepal and North Dakota is available. Spot blotch is one of the most devastating diseases of wheat in Nepal and causes up to $30 \%$ yield loss (12). In addition, Nepal's environment is conducive for the occurrence of spot blotch epidemics and, thus, the International Maize and Wheat Improvement Center has chosen it as one of the primary spot blotch screening sites $(21,39-41,43)$. Therefore, it is imperative to know about phenotypic and molecular variations in $C$. sativus in this region where wheat is planted in both hills (elevation of 800 to 4,000 m, with subtropical climate) and plains in Nepal (elevation of 75 to $1,000 \mathrm{~m}$, with tropical climate). The climatic conditions and cultural practices for wheat cultivation in Nepal are quite distinct from North Dakota. Wheat cultivars grown in Nepal are mostly either introduced from other countries or released by the National Wheat Research Program (NWRP), Bhairahawa, Nepal. In contrast, North Dakota has a temperate climate and is one of the largest wheat producing states in the United States (47), where spot blotch has recently emerged as an important foliar disease (46). Wheat cultivars in North Dakota are exposed to great differences in weather conditions and length of host-free periods, and these cultivars have various sources of spot blotch resistance incorporated by wheat breeding programs (19). In addition, the spring wheat cultivars are planted in the spring and harvested during late summer or early fall whereas the winter wheat cultivars are sown in the fall, remain dormant during the winter, and are harvested during June or July. C. sativus populations infecting spring wheat survive a host-free period of extremely cold winter weather, whereas those on winter wheat survive in a host-free period of hot and dry weather during summer 
and early fall (19). We hypothesize that populations of $C$. sativus in the Hill and Plain areas in Nepal and in the Central and Northern areas of North Dakota might evolve high virulence and could be genetically diverse. The main objectives of this study were to determine virulence in $C$. sativus populations from wheat in Nepal and North Dakota on differential wheat lines and cultivars and examine their genetic variation using selected polymorphic amplified fragment length polymorphism (AFLP) primers. Given the lack of knowledge of pathogenic and genetic diversity of $C$. sativus from wheat, this work will provide descriptive data to fill in an important information gap.

\section{Materials and Methods}

Fungal isolates. The isolates from Nepal were collected from major wheat-producing areas in the hilly production areas of the Kathmandu, Lalitpur, Bhaktapur, Pokhara, Kavreplanchowk, Solukhumbu, Parbat, Baglung, and Dhankuta districts and in the Plain areas from the Sunsari, Morang, Dhanusha, Bara, Parsa, Chitwan, Rupandehi, Banke, Bardiya, and Kanchanpur districts during 2009 (Table 1). Wheat leaves showing typical spot blotch symptoms were collected randomly from wheat fields in Central and Northern areas of North Dakota during 2006 and 2007 (Table 1). Singlespore isolation of $C$. sativus from the Central and Northern areas of North Dakota was performed by S. Ali at North Dakota State University (NDSU), Fargo (Table 1). Single-spore isolation of $C$. sativus from the Hill and Plain areas of Nepal was done at the Division of Plant Pathology, Nepal Agriculture Research Council, Khumaltar, Nepal and brought to NDSU under United States Department of Agriculture Animal and Plant Health Inspection Service permit number P526P-09-02536.

For isolation, diseased leaf samples were cut into 2- to 3-mm segments, placed in a plate with $0.5 \%$ sodium hypochlorite solution for $30 \mathrm{~s}$, and later rinsed twice with sterile distilled water. Leaf segments were dried on sterile Whatman filter paper in a laminar flow hood and later placed in moist filter paper and kept in an incubator for 24 to $48 \mathrm{~h}$ at $21^{\circ} \mathrm{C}$. The leaves were then observed under the stereoscope and a single conidium was picked using a flamesterilized steel needle. Each single conidium was transferred to a petri dish containing V8-potato dextrose agar (PDA) (150 ml of V8 juice, $10 \mathrm{~g}$ of Difco PDA, $3 \mathrm{~g}$ of $\mathrm{CaCo}_{3}, 10 \mathrm{~g}$ of Bacto agar, and $850 \mathrm{ml}$ of distilled water) and incubated at $21^{\circ} \mathrm{C}$ for 8 to 10 days under continuous light condition. At least two subsequent singlespore transfers were made to ensure the purity of each isolate. Later, the single-spore isolates from each V8-PDA plate were cut into small blocks, air dried under a laminar flow hood for $24 \mathrm{~h}$, and stored at $-80^{\circ} \mathrm{C}$. In total, 96 putative isolates of $C$. sativus from the Hill $(n=24)$ and Plain $(n=24)$ areas in Nepal and from the Central $(n=24)$ and Northern $(n=24)$ areas in North Dakota and were used in this study (Table 1).

Virulence analysis. Twelve differential wheat lines (Table 2) originating from Brazil, China, India, and Nepal (22,24,25,33, 34,41-43) with different levels of resistance to C. sativus were selected based on differential responses in previous studies $(21,29)$. Ten of these wheat lines have exhibited resistance to $C$. sativus under field conditions and have been used as potential sources of resistance to $C$. sativus in wheat breeding programs in Asia $(24,25,33,40-44)$. Two differential wheat lines (Sonalika and BL 1473) were included as susceptible checks.

In total, 95 isolates of $C$. sativus (Central $[n=24]$ and Northern $[n=24]$ areas of North Dakota and Hill $[n=24]$ and Plain $[n=23]$ areas of Nepal) were evaluated for their virulence on the 12 differential wheat lines. Pathogen inoculation was conducted under controlled conditions in growth chambers (16). Each experiment was conducted using a randomized complete block design with three replications. Each experimental unit had three seeds of a cultivar planted in a plastic "conetainer" $(3.8 \mathrm{~cm}$ in diameter and $20 \mathrm{~cm}$ long) filled with Fison sunshine Mix number 1 (Fison Horticulture). Each plant in the conetainer was considered a sampling unit. The conetainers were placed in RL98 trays (Stuewe and Sons, Inc.). For each cultivar, nine plants were used and tested for each isolate. In each experiment, wheat lines were considered a fixed effect and isolate was considered a random effect. The experiment was repeated once.

Inoculum of each isolate was prepared as described previously by Mahto et al. (30). The conidial suspension was adjusted to $3.5 \times 10^{3}$ conidia/ml and conidia were counted using a hemacytometer. One drop of Tween 20 per $100 \mathrm{ml}$ of spore suspension was added as a surfactant. Two-week-old seedlings at Zadoks growth stage 13 (55) were spray inoculated with the spore suspension until run-off. Following inoculation, seedlings were moved into a mist chamber to provide continuous leaf wetness. After $24 \mathrm{~h}$, seedlings were moved to a growth chamber at 21 and $18^{\circ} \mathrm{C}$ (day and night, respectively) with a 16-h photoperiod. Disease scores were assessed on second leaves between 9 and 10 days after inoculation using a 1-to-9 scale which was developed for scoring $C$. sativus infection response in barley (16). However, disease scores from 1 to 4 were considered resistant $(\mathrm{R})$ because they had minute to small round or oblong necrotic lesions $\left(<2 \mathrm{~mm}^{2}\right)$ with or without restricted chlorotic margins. Similarly, disease reactions $>4$ were considered susceptible (S) because they had medium to large elliptical or ovate necrotic lesions $\left(>2 \mathrm{~mm}^{2}\right)$ with chlorotic margins and expanding diffuse chlorosis.

Virulence and cluster analysis. The term "virulence" is defined as the ability of an isolate to cause disease by overcoming a specific host $R$ gene and "pathogenicity" as both the specific and nonspecific disease-causing abilities of a pathogen (49). However, herein, we have used the term "virulence group" as the ability of a group of isolates to cause disease across a set of 12 differential wheat lines as previously mentioned by Ghazvini and Tekauz (17). Similarly, the term "pathotype" was used to describe levels of aggressiveness of isolates with a smaller set of differential genotypes for $C$. sativus $(17,23)$, and Pyrenophora teres f. teres from barley $(7,28)$. Nonparametric analysis $(39)$ was performed using disease scores obtained from each cultivar-isolate combination from each experiment. The median values were estimated using the SAS program PROC Mixed (version 9.2; SAS Institute) (38). To test whether the median disease scores of both experiments were similar, the interactions between the wheat lines and the fungal isolates and between two experiments were calculated (1). To test the homogeneity of variances among the two experiments, Bartlett's $\chi^{2}$ values were estimated using SAS as described previously (1).

The reactions of 12 wheat differential lines to each of the $95 C$. sativus isolates were shown by preparing a resistance/susceptibility (R/S) matrix (Table 3). The triplet coded nomenclature system, as proposed by Limpert and Muller (27), was also used to designate the virulence group of the 95 isolates of $C$. sativus. This system has been used to classify virulence group of $C$. sativus previously $(17,18)$. Hierarchical cluster analysis was performed using the SIMINT program in the NTSYS-pc software (version 2.1; Exeter Software) in order to evaluate the intra- and inter-relationships among isolates of $C$. sativus in each pathotype. Cluster analysis was performed using the median disease rating score of differential wheat lines to each isolate $(18,23)$.

Genomic DNA extraction and quantification. To extract genomic DNA, each isolate was grown on V8-PDA plates overlaid with cellophane sheets, and mycelia grown on the cellophane surface were scraped directly with a sterile scalpel and harvested from each plate after 15 days. Harvested mycelia were placed in a 2-ml centrifuge tube and freeze dried at $-80^{\circ} \mathrm{C}$ for $48 \mathrm{~h}$. The lyophilized mycelia were ground to fine powder using a high-speed mixer mill (Model MM301; Retsch Inc.). Genomic DNA of each isolate (total of 96 isolates; 48 isolates each from Nepal and North Dakota) was extracted using a DNeasy Plant Mini Kit (Qiagen Inc.) and was quantified using a Nano Drop (Model ND-1000; Thermo Scientific Inc.). Each DNA sample was adjusted to $30 \mathrm{ng} / \mu \mathrm{l}$ for AFLP analysis.

Sequence analysis. To confirm the identity of $C$. sativus isolates, we sequenced and compared internal transcribed spacer (ITS) 4 and ITS5 and glyceraldehyde-3-phosphate dehydrogenase $(g p d)$ regions from the 96 isolates of $C$. sativus. ITS and gpd sequences were further compared with ITS and gpd sequences 
Table 1. Cochliobolus sativus isolates collected from wheat in Hill and Plain areas in Nepal and Central and Northern areas in North Dakota (ND) which were used for pathogenic and molecular diversity studies

\begin{tabular}{|c|c|c|c|c|c|}
\hline Serial number & Isolate designation & Date of collection & Cultivar or wheat type $\mathrm{a}^{\mathrm{a}}$ & Origin & Region \\
\hline 1 & $\mathrm{CS} 1$ & 2009 & Spring wheat & Solukhumbu & Hill \\
\hline 2 & $\mathrm{CS} 2$ & 2009 & Spring wheat & Solukhumbu & Hill \\
\hline 3 & CS3 & 2009 & Spring wheat & Dhankuta & Hill \\
\hline 4 & $\mathrm{CS} 4$ & 2009 & Spring wheat & Dhankuta & Hill \\
\hline 5 & CS5 & 2009 & Spring wheat & Dhankuta & Hill \\
\hline 6 & CS6 & 2009 & Spring wheat & Kavrepalanchowk & Hill \\
\hline 7 & CS7 & 2009 & Spring wheat & Kavrepalanchowk & Hill \\
\hline 8 & CS8 & 2009 & Spring wheat & Kavrepalanchowk & Hill \\
\hline 9 & CS9 & 2009 & Spring wheat & Kavrepalanchowk & Hill \\
\hline 10 & CS10 & 2009 & Spring wheat & Kavrepalanchowk & Hill \\
\hline 11 & CS11 & 2009 & Spring wheat & Bhaktapur & Hill \\
\hline 12 & $\mathrm{CS} 12$ & 2009 & Spring wheat & Bhaktapur & Hill \\
\hline 13 & CS13 & 2009 & Spring wheat & Bhaktapur & Hill \\
\hline 14 & CS14 & 2009 & Spring wheat & Bhaktapur & Hill \\
\hline 15 & CS15 & 2009 & Spring wheat & Bhaktapur & Hill \\
\hline 16 & CS16 & 2009 & Spring wheat & Kathmandu & Hill \\
\hline 17 & $\mathrm{CS} 17$ & 2009 & Spring wheat & Kathmandu & Hill \\
\hline 18 & CS18 & 2009 & Spring wheat & Kathmandu & Hill \\
\hline 19 & CS19 & 2009 & Spring wheat & Kathmandu & Hill \\
\hline 20 & CS20 & 2009 & Spring wheat & Kathmandu & Hill \\
\hline 21 & CS21 & 2009 & Spring wheat & Kathmandu & Hill \\
\hline 22 & CS22 & 2009 & Spring wheat & Lalitpur & Hill \\
\hline 23 & $\mathrm{CS} 23$ & 2009 & Spring wheat & Baglung & Hill \\
\hline 24 & $\mathrm{CS} 24$ & 2009 & Spring wheat & Parbat & Hill \\
\hline 25 & CS25 & 2009 & Spring wheat & Morang & Plain \\
\hline 26 & $\mathrm{CS} 26$ & 2009 & Spring wheat & Morang & Plain \\
\hline 27 & CS27 & 2009 & Spring wheat & Morang & Plain \\
\hline 28 & CS28 & 2009 & Spring wheat & Morang & Plain \\
\hline 29 & CS30 & 2009 & Spring wheat & Sunsari & Plain \\
\hline 30 & CS31 & 2009 & Spring wheat & Sunsari & Plain \\
\hline 31 & CS32 & 2009 & Spring wheat & Sunsari & Plain \\
\hline 32 & CS33 & 2009 & Spring wheat & Janakpur & Plain \\
\hline 33 & CS34 & 2009 & Spring wheat & Janakpur & Plain \\
\hline 34 & CS35 & 2009 & Spring wheat & Janakpur & Plain \\
\hline 35 & CS36 & 2009 & Spring wheat & Bara & Plain \\
\hline 36 & CS37 & 2009 & Spring wheat & Bara & Plain \\
\hline 37 & CS38 & 2009 & Spring wheat & Bara & Plain \\
\hline 38 & CS39 & 2009 & Spring wheat & Parsa & Plain \\
\hline 39 & CS40 & 2009 & Spring wheat & Parsa & Plain \\
\hline 40 & CS41 & 2009 & Spring wheat & Parsa & Plain \\
\hline 41 & CS42 & 2009 & Spring wheat & Chitwan & Plain \\
\hline 42 & CS43 & 2009 & Spring wheat & Chitwan & Plain \\
\hline 43 & CS44 & 2009 & Spring wheat & Chitwan & Plain \\
\hline 44 & CS45 & 2009 & Spring wheat & Rupandehi & Plain \\
\hline 45 & CS46 & 2009 & Spring wheat & Rupandehi & Plain \\
\hline 46 & CS47 & 2009 & Spring wheat & Rupandehi & Plain \\
\hline 47 & CS48 & 2009 & Spring wheat & Banke & Plain \\
\hline 48 & CS49 & 2009 & Spring wheat & Banke & Plain \\
\hline 49 & CS50 & 2007 & Goodstreak (HRWW) & Bottineau, ND & Northern \\
\hline 50 & CS51 & 2006 & Spring wheat & Bottineau, ND & Northern \\
\hline 51 & CS52 & 2007 & Wesley (WW) & Lisbon, ND & Central \\
\hline 52 & CS53 & 2006 & Spring wheat & Griggs, ND & Northern \\
\hline 53 & CS54 & 2006 & Spring wheat & Griggs, ND & Northern \\
\hline 54 & CS55 & 2006 & Spring wheat & Griggs, ND & Northern \\
\hline 55 & CS56 & 2006 & Spring wheat & Nelson, ND & Northern \\
\hline 56 & CS57 & 2006 & Spring wheat & Towner, ND & Northern \\
\hline 57 & CS58 & 2006 & Spring wheat & Bottineau, ND & Northern \\
\hline 58 & CS59 & 2007 & Wesley (WW) & Lisbon, ND & Central \\
\hline 59 & CS60 & 2006 & Spring wheat & Bottineau, ND & Northern \\
\hline 60 & CS61 & 2007 & Spring wheat & Minot, ND & Northern \\
\hline 61 & CS62 & 2007 & SD 96240-3-1 (WW) & Lisbon, ND & Central \\
\hline 62 & CS63 & 2007 & Jerry (WW) & Prosper, ND & Central \\
\hline 63 & CS64 & 2007 & SD 96240-3-1 (WW) & Lisbon, ND & Central \\
\hline 64 & CS65 & 2007 & Millennium (WW) & Lisbon, ND & Central \\
\hline 65 & CS66 & 2006 & Spring wheat & Carrington, ND & Central \\
\hline 66 & CS67 & 2007 & SD 96240-3-1 (WW) & Lisbon, ND & Central \\
\hline 67 & CS68 & 2006 & Spring wheat & Carrington, ND & Central \\
\hline 68 & CS69 & 2007 & Goodstreak (HRWW) & Prosper, ND & Central \\
\hline 69 & CS70 & 2007 & Darrel (HRWW) & Lisbon, ND & Central \\
\hline 70 & CS71 & 2006 & Spring wheat & Griggs, ND & Northern \\
\hline 71 & $\mathrm{CS} 72$ & 2006 & Spring wheat & Griggs, ND & Northern \\
\hline \multirow[t]{2}{*}{72} & CS73 & 2007 & Goodstreak (HRWW) & Prosper, ND & Central \\
\hline & & & & & (continued on next page) \\
\hline
\end{tabular}

${ }^{\mathrm{a}} \mathrm{HRWW}=$ hard red winter wheat, $\mathrm{HRSW}=$ hard red spring wheat, $\mathrm{SW}=$ spring wheat, and $\mathrm{WW}=$ winter wheat. 
Table 1. (continued from preceding page)

\begin{tabular}{|c|c|c|c|c|c|}
\hline Serial number & Isolate designation & Date of collection & Cultivar or wheat type ${ }^{a}$ & Origin & Region \\
\hline 73 & CS74 & 2006 & Spring wheat & Ramsey, ND & Northern \\
\hline 74 & CS75 & 2007 & Wesley (WW) & Lisbon, ND & Central \\
\hline 75 & CS76 & 2007 & Alsen (SW) & Langdon, ND & Northern \\
\hline 76 & CS77 & 2006 & Spring wheat & Ramsey, ND & Northern \\
\hline 77 & CS78 & 2006 & Spring wheat & Nelson, ND & Northern \\
\hline 78 & CS79 & 2007 & Goodstreak (HRWW) & Prosper, ND & Central \\
\hline 79 & CS80 & 2006 & Spring wheat & Towner, ND & Northern \\
\hline 80 & CS81 & 2007 & SD 96240-3-1 (WW) & Lisbon, ND & Central \\
\hline 81 & CS82 & 2006 & Spring wheat & Carrington, ND & Central \\
\hline 82 & CS83 & 2006 & Spring wheat & Prosper, ND & Central \\
\hline 83 & CS84 & 2007 & Darrel (HRWW) & Lisbon, ND & Central \\
\hline 84 & CS85 & 2006 & Spring wheat & Nelson, ND & Northern \\
\hline 85 & CS86 & 2006 & Spring wheat & Nelson, ND & Northern \\
\hline 86 & CS87 & 2006 & Spring wheat & Rolette, ND & Northern \\
\hline 87 & CS88 & 2006 & Spring wheat & Rolette, ND & Northern \\
\hline 88 & CS89 & 2006 & Spring wheat & Rolette, ND & Northern \\
\hline 89 & CS90 & 2007 & Alice (HRWW) & Lisbon, ND & Central \\
\hline 90 & CS91 & 2007 & Darrel (HRWW) & Lisbon, ND & Central \\
\hline 91 & CS92 & 2006 & Spring wheat & Walsh, ND & Northern \\
\hline 92 & CS93 & 2007 & CDC Falcon (WW) & Lisbon, ND & Central \\
\hline 93 & CS94 & 2007 & Wesley (WW) & Lisbon, ND & Central \\
\hline 94 & CS95 & 2006 & Spring wheat & Prosper, ND & Central \\
\hline 95 & CS96 & 2007 & Howard (HRSW) & Langdon, ND & Northern \\
\hline 96 & CS97 & 2007 & Wesley (WW) & Lisbon, ND & Central \\
\hline
\end{tabular}

Table 2. Differential wheat lines used to assess virulence of Cochliobolus sativus isolates from wheat in Nepal and North Dakota

\begin{tabular}{|c|c|c|c|c|}
\hline Genotype & Levela & Pedigree & $\begin{array}{c}\text { CIMMYT } \\
\text { CID }^{\mathbf{b}}\end{array}$ & Origin \\
\hline BL $1473^{c}$ & $\mathrm{~S}$ & Nepal 297/NL531 & 166468 & Nepal/CIMMYT \\
\hline Chirya $7^{\mathrm{d}}$ & $\mathrm{R}$ & CS/Th. cu//Glen/3/Ald/Pvn/4/Ningmai No.4/Oleson//Ald/Yangmai No.4 & 66176 & Nepal/CIMMYT \\
\hline Chirya $3^{\text {c,e }}$ & $\mathrm{R}$ & CS/Th. cu//Vee/3/Ald/Pvn/4/Ningmai No.4/Oleson//Ald/Yangmai No.4 & 54384 & CIMMYT \\
\hline $\mathrm{G} 162^{\mathrm{f}}$ & $\mathrm{R}$ & Guizhou large head no.7//Yangmai-1/Op & 93926 & China \\
\hline Milan/Shanghai $7^{\mathrm{c}}$ & $\mathrm{R}$ & VS73.600/MIRLO/3/BOW//YE/TRF/4/Shanghai7 & 20026 & China \\
\hline Ning $8201^{\mathrm{e}}$ & $\mathrm{R}$ & Ningmai-4/Olesen/Alondra (Sib)/Yangmai-3 & 95659 & China \\
\hline Ning $8319^{g}$ & $\mathrm{R}$ & Ningmai-4/Oleson//Ald/Yangmai-3 & 3495 & China \\
\hline OCEPAR- $7^{\mathrm{h}}$ & $\mathrm{R}$ & Tzpp*2/An64//Inia66/3/Cno67/Jar//Kvz & 7136 & Brazil \\
\hline PBW $343^{\mathrm{i}}$ & $\mathrm{R}$ & Nd/VG9144//Kal/Bb/3/Yaco/4/Vee5 & 8890 & India \\
\hline Sonalika $^{\mathrm{j}}$ & $\mathrm{S}$ & II53.388/AN//YT54/N10B/3/LR/4/B4946/Y53//3*Y50 & 6977 & Nepal/CIMMYT \\
\hline SW89.5422g & $\mathrm{R}$ & Unknown & 72403 & China \\
\hline Yangmai- $6^{\mathrm{i}}$ & $\mathrm{R}$ & Unknown & 239288 & China \\
\hline
\end{tabular}

a Level of field resistance according to evaluation of lesion density (lesions $\mathrm{cm}^{2}$ ) and area under disease progress curve of seedling and adult plant resistance to spot blotch. $\mathrm{S}=$ susceptible and $\mathrm{R}=$ resistant.

${ }^{\mathrm{b}}$ International Maize and Wheat Improvement Center (CIMMYT) cross identification number (CID).

c Neupane et al. (33).

d Sharma et al. (42).

e Kumar et al. (25).

${ }^{\mathrm{f}}$ Sharma et al. (43).

g Sharma et al. (41)

${ }^{\mathrm{h}}$ Okuyama et al. (34).

i Joshi et al. (22).

j Kumar et al. (24).

reported from closely related species, including C. carbonum (gpd $=$ GenBank accession AF081382; ITS = AF158110), C. victoriae ( gpd $=\mathrm{AF} 158112 ;$ ITS $=\mathrm{AF} 158109)$, C. heterostrophus $(g p d=$ AF081380; ITS $=$ AF071325), and C. eleusines $(g p d=\mathrm{AF081405}$; ITS $=$ AF081451) (data not shown). The ITS regions were amplified using the standard primers ITS4 and ITS5 (51) and the region coding for the nuclear gene gpd was amplified using the primer pair gpdl/gpd2 (6) and, for both ITS and gpd sequence amplifications, polymerase chain reaction (PCR) cycles and conditions were similar to those described previously by Berbee et al. (6). For confirmation, $5 \mu \mathrm{l}$ of the amplified PCR products were run in $2 \%$ (wt/vol) agarose gel (Sigma-Aldrich) in $0.5 \times$ Tris-borate-EDTA (TBE) buffer (0.089 M Tris-borate, $0.089 \mathrm{M}$ boric acid, and 0.002 M EDTA). After confirming the PCR reaction, the remaining $20 \mu \mathrm{l}$ of the amplified products were sent to the Molecular Cloning Laboratories DNA sequencing service (San Francisco, CA) for sequencing of ITS and gpd genomic regions in both sense and antisense directions. Sequences derived in this study were compared with the ITS and $g p d$ sequence with other closely related species sequence after downloading the sequences from GenBank (http://www.ncbi.nlm.nih.gov/BLAST). Both the ITS and gpd sequences were aligned using the software ClustalX and neighborjoining trees, with 1,000 bootstraps built to obtain the phylogenetic trees.

AFLP analysis. AFLP analysis was performed using an AFLP core reagent kit (Invitrogen Life Technologies) and reactions were conducted as described previously (50). Genomic DNA (30 ng/ $\mu \mathrm{l})$ of each isolate was digested with EcoRI and $\mathrm{MseI}$ at $37^{\circ} \mathrm{C}$ for $3 \mathrm{~h}$ and, later, the restricted DNA fragments were ligated to AFLP adaptors at $20^{\circ} \mathrm{C}$ for $3 \mathrm{~h}$. The digested and ligated templates were diluted in 1:2 ratios with sterile distilled water before being used in the preamplification. Primers complimentary to the adapter sequences plus one selective base at the $3^{\prime}$ end were used for preamplifications. All the samples were preamplified in reactions containing the DNA template $(30 \mathrm{ng} / \mu \mathrm{l}), 36 \mathrm{ng}$ of each EcoRI primer (E-A) and MseI primer (M-C), $0.2 \mathrm{mM}$ dNTPs, $1 \times$ buffer, 
Table 3. Median disease values of 95 isolates of Cochliobolus sativus on 12 differential wheat lines and corresponding pathotypes and virulence types based on growth chamber studies ${ }^{\mathrm{a}}$

\begin{tabular}{|c|c|c|c|c|c|c|c|c|c|c|c|c|c|c|c|}
\hline Isolate & BL 1473 & Chirya-7 & Chirya-3 & G 162 & MS-7 & $\begin{array}{l}\text { Ning } \\
8201\end{array}$ & $\begin{array}{l}\text { Ning } \\
8319\end{array}$ & Ocepar-7 & PBW 343 & Sonalika & $\begin{array}{c}\text { SW 89- } \\
5422\end{array}$ & Yangmai-6 & Code $^{b}$ & Group $^{c}$ & Pathotype $^{\mathrm{d}}$ \\
\hline Cs 51 & $\mathrm{R}$ & $\mathrm{R}$ & $\mathrm{R}$ & $\mathrm{R}$ & $\mathrm{R}$ & $\mathrm{R}$ & $\mathrm{R}$ & $\mathrm{R}$ & $\mathrm{R}$ & $\mathrm{R}$ & $\mathrm{R}$ & $\mathrm{R}$ & 0.0 .0 .0 & $\mathrm{~L}$ & 1 \\
\hline Cs 82 & $\mathrm{R}$ & $\mathrm{R}$ & $\mathrm{R}$ & $\mathrm{R}$ & $\mathrm{R}$ & $\mathrm{R}$ & $\mathrm{R}$ & $\mathrm{R}$ & $\mathrm{R}$ & $\mathrm{R}$ & $\mathrm{R}$ & $\mathrm{R}$ & 0.0 .0 .0 & $\mathrm{~L}$ & 1 \\
\hline Cs 78 & $\mathrm{R}$ & $\mathrm{R}$ & $\mathrm{R}$ & $\mathrm{R}$ & $\mathrm{R}$ & $\mathrm{R}$ & $\mathrm{R}$ & $\mathrm{R}$ & $\mathrm{R}$ & $\mathrm{R}$ & $\mathrm{R}$ & $\mathrm{R}$ & 0.0 .0 .0 & $\mathrm{~L}$ & 1 \\
\hline Cs 4 & $\mathrm{R}$ & $\mathrm{R}$ & $\mathrm{R}$ & $\mathrm{R}$ & $\mathrm{R}$ & $\mathrm{R}$ & $\mathrm{R}$ & $\mathrm{R}$ & $\mathrm{R}$ & $\mathrm{R}$ & $\mathrm{R}$ & $\mathrm{R}$ & 0.0 .0 .0 & $\mathrm{~L}$ & 1 \\
\hline Cs 93 & $\mathrm{R}$ & $\mathrm{R}$ & $\mathrm{R}$ & $\mathrm{R}$ & $\mathrm{R}$ & $\mathrm{R}$ & $\mathrm{R}$ & $\mathrm{R}$ & $\mathrm{R}$ & $\mathrm{R}$ & $\mathrm{R}$ & $\mathrm{R}$ & 0.0 .0 .0 & $\mathrm{~L}$ & 1 \\
\hline Cs 16 & $\mathrm{R}$ & $\mathrm{R}$ & $\mathrm{R}$ & $\mathrm{R}$ & $\mathrm{R}$ & $\mathrm{R}$ & $\mathrm{R}$ & $\mathrm{R}$ & $\mathrm{R}$ & $\mathrm{R}$ & $\mathrm{R}$ & $\mathrm{R}$ & 0.0 .0 .0 & $\mathrm{~L}$ & 1 \\
\hline Cs 2 & $\mathrm{R}$ & $\mathrm{R}$ & $\mathrm{R}$ & $\mathrm{R}$ & $\mathrm{R}$ & $\mathrm{R}$ & $\mathrm{R}$ & $\mathrm{R}$ & $\mathrm{R}$ & $\mathrm{R}$ & $\mathrm{R}$ & $\mathrm{R}$ & 0.0 .0 .0 & $\mathrm{~L}$ & 1 \\
\hline Cs 12 & $\mathrm{R}$ & $\mathrm{R}$ & $\mathrm{R}$ & $\mathrm{R}$ & $\mathrm{R}$ & $\mathrm{R}$ & $\mathrm{R}$ & $\mathrm{R}$ & $\mathrm{R}$ & $\mathrm{R}$ & $\mathrm{R}$ & $\mathrm{R}$ & 0.0 .0 .0 & $\mathrm{~L}$ & 1 \\
\hline Cs 65 & $\mathrm{R}$ & $\mathrm{R}$ & $\mathrm{R}$ & $\mathrm{R}$ & $\mathrm{R}$ & $\mathrm{R}$ & $\mathrm{R}$ & $\mathrm{R}$ & $\mathrm{R}$ & $\mathrm{R}$ & $\mathrm{R}$ & $\mathrm{R}$ & 0.0 .0 .0 & $\mathrm{~L}$ & 1 \\
\hline Cs 90 & $\mathrm{R}$ & $\mathrm{R}$ & $\mathrm{R}$ & $\mathrm{R}$ & $\mathrm{R}$ & $\mathrm{R}$ & $\mathrm{R}$ & $\mathrm{R}$ & $\mathrm{R}$ & $\mathrm{S}$ & $\mathrm{R}$ & $\mathrm{R}$ & 0.0 .0 .1 & I & 2 \\
\hline Cs 64 & $\mathrm{R}$ & $\mathrm{R}$ & $\mathrm{R}$ & $\mathrm{R}$ & $\mathrm{R}$ & $\mathrm{R}$ & $\mathrm{R}$ & $\mathrm{R}$ & $\mathrm{R}$ & $S$ & $\mathrm{R}$ & $\mathrm{R}$ & 0.0 .0 .1 & I & 2 \\
\hline Cs 21 & $\mathrm{R}$ & $\mathrm{R}$ & $\mathrm{R}$ & $\mathrm{R}$ & $\mathrm{R}$ & $\mathrm{R}$ & $\mathrm{R}$ & $\mathrm{S}$ & $\mathrm{R}$ & $\mathrm{S}$ & $\mathrm{R}$ & $\mathrm{R}$ & 0.0 .2 .1 & I & 3 \\
\hline Cs 36 & $\mathrm{R}$ & $\mathrm{R}$ & $\mathrm{R}$ & $\mathrm{R}$ & $\mathrm{R}$ & $\mathrm{R}$ & $\mathrm{R}$ & $\mathrm{S}$ & $\mathrm{R}$ & $\mathrm{S}$ & $\mathrm{S}$ & $\mathrm{R}$ & 0.0 .2 .3 & I & 4 \\
\hline Cs 1 & $\mathrm{R}$ & $\mathrm{R}$ & $\mathrm{R}$ & $\mathrm{R}$ & $\mathrm{R}$ & $\mathrm{R}$ & $\mathrm{R}$ & $\mathrm{R}$ & $\mathrm{S}$ & $\mathrm{S}$ & $\mathrm{R}$ & $\mathrm{R}$ & 0.0 .4 .1 & I & 5 \\
\hline Cs 30 & $\mathrm{R}$ & $\mathrm{R}$ & $\mathrm{R}$ & $\mathrm{R}$ & $\mathrm{R}$ & $\mathrm{R}$ & $\mathrm{R}$ & $\mathrm{R}$ & $\mathrm{S}$ & $\mathrm{S}$ & $\mathrm{R}$ & $\mathrm{R}$ & 0.0 .4 .1 & I & 5 \\
\hline Cs 92 & $\mathrm{R}$ & $\mathrm{R}$ & $\mathrm{R}$ & $\mathrm{R}$ & $\mathrm{R}$ & $\mathrm{R}$ & $\mathrm{R}$ & $\mathrm{R}$ & $\mathrm{S}$ & $\mathrm{S}$ & $\mathrm{R}$ & $\mathrm{R}$ & 0.0 .4 .1 & I & 5 \\
\hline Cs 59 & $\mathrm{R}$ & $\mathrm{R}$ & $\mathrm{R}$ & $\mathrm{S}$ & $\mathrm{R}$ & $\mathrm{R}$ & $\mathrm{R}$ & $\mathrm{R}$ & $\mathrm{R}$ & $\mathrm{S}$ & $\mathrm{R}$ & $\mathrm{R}$ & 0.1 .0 .1 & I & 6 \\
\hline Cs 9 & $\mathrm{R}$ & $\mathrm{R}$ & $\mathrm{R}$ & $S$ & $\mathrm{R}$ & $\mathrm{R}$ & $\mathrm{R}$ & $\mathrm{R}$ & $\mathrm{R}$ & $S$ & $\mathrm{R}$ & $\mathrm{R}$ & 0.1 .0 .1 & I & 6 \\
\hline Cs 96 & $\mathrm{R}$ & $\mathrm{R}$ & $\mathrm{R}$ & $\mathrm{S}$ & $\mathrm{R}$ & $\mathrm{R}$ & $\mathrm{R}$ & $\mathrm{R}$ & $\mathrm{S}$ & $\mathrm{S}$ & $\mathrm{R}$ & $\mathrm{R}$ & 0.1 .4 .1 & I & 7 \\
\hline Cs 73 & $\mathrm{~S}$ & $\mathrm{R}$ & $\mathrm{R}$ & $\mathrm{R}$ & $\mathrm{R}$ & $\mathrm{R}$ & $\mathrm{R}$ & $\mathrm{R}$ & $\mathrm{R}$ & $\mathrm{R}$ & $\mathrm{R}$ & $\mathrm{R}$ & 1.0 .0 .0 & I & 8 \\
\hline Cs 87 & $\mathrm{R}$ & $\mathrm{R}$ & $\mathrm{R}$ & $\mathrm{S}$ & $\mathrm{R}$ & $\mathrm{R}$ & $\mathrm{R}$ & $\mathrm{S}$ & $\mathrm{S}$ & $\mathrm{S}$ & $\mathrm{R}$ & $\mathrm{R}$ & 0.1 .6 .1 & I & 9 \\
\hline Cs 88 & $\mathrm{R}$ & $\mathrm{R}$ & $\mathrm{R}$ & $\mathrm{R}$ & $\mathrm{S}$ & $\mathrm{R}$ & $\mathrm{R}$ & $\mathrm{S}$ & $\mathrm{S}$ & $\mathrm{S}$ & $\mathrm{R}$ & $\mathrm{R}$ & 0.2 .6 .1 & I & 10 \\
\hline Cs 26 & $\mathrm{~S}$ & $\mathrm{R}$ & $\mathrm{R}$ & $\mathrm{R}$ & $\mathrm{R}$ & $\mathrm{R}$ & $\mathrm{R}$ & $\mathrm{R}$ & $\mathrm{R}$ & $\mathrm{S}$ & $\mathrm{R}$ & $\mathrm{S}$ & 1.0 .0 .5 & I & 11 \\
\hline Cs 62 & $\mathrm{~S}$ & $\mathrm{R}$ & $\mathrm{R}$ & $\mathrm{R}$ & $\mathrm{R}$ & $\mathrm{R}$ & $\mathrm{R}$ & $\mathrm{R}$ & $\mathrm{S}$ & $\mathrm{S}$ & $\mathrm{R}$ & $\mathrm{S}$ & 1.0 .4 .5 & I & 12 \\
\hline Cs 63 & $S$ & $\mathrm{R}$ & $\mathrm{R}$ & $\mathrm{R}$ & $\mathrm{R}$ & $\mathrm{R}$ & $\mathrm{R}$ & $\mathrm{S}$ & $\mathrm{S}$ & $\mathrm{S}$ & $\mathrm{R}$ & $\mathrm{R}$ & 1.0 .6 .1 & I & 13 \\
\hline Cs 52 & $\mathrm{~S}$ & $\mathrm{R}$ & $\mathrm{R}$ & $\mathrm{R}$ & $\mathrm{R}$ & $\mathrm{R}$ & $\mathrm{R}$ & $\mathrm{S}$ & $\mathrm{S}$ & $\mathrm{S}$ & $\mathrm{R}$ & $\mathrm{R}$ & 1.0 .6 .1 & I & 13 \\
\hline Cs 6 & $\mathrm{~S}$ & $\mathrm{R}$ & $\mathrm{R}$ & $\mathrm{S}$ & $\mathrm{R}$ & $\mathrm{R}$ & $\mathrm{R}$ & $\mathrm{S}$ & $\mathrm{R}$ & $\mathrm{S}$ & $\mathrm{R}$ & $\mathrm{R}$ & 1.1.2.1 & I & 14 \\
\hline Cs 8 & $\mathrm{~S}$ & $\mathrm{R}$ & $\mathrm{R}$ & $\mathrm{S}$ & $\mathrm{R}$ & $\mathrm{R}$ & $\mathrm{R}$ & $\mathrm{S}$ & $\mathrm{R}$ & $\mathrm{S}$ & $\mathrm{R}$ & $\mathrm{R}$ & 1.1.2.1 & I & 14 \\
\hline Cs 7 & $\mathrm{~S}$ & $\mathrm{R}$ & $\mathrm{R}$ & S & $\mathrm{R}$ & $\mathrm{R}$ & $\mathrm{R}$ & S & $\mathrm{R}$ & S & $\mathrm{S}$ & $\mathrm{R}$ & 1.1 .2 .3 & I & 15 \\
\hline Cs10 & $S$ & $\mathrm{R}$ & $\mathrm{R}$ & $\mathrm{S}$ & $\mathrm{R}$ & $\mathrm{R}$ & $\mathrm{R}$ & $\mathrm{S}$ & $\mathrm{R}$ & $\mathrm{S}$ & $\mathrm{S}$ & $\mathrm{S}$ & 1.1 .2 .7 & I & 16 \\
\hline Cs 74 & $\mathrm{~S}$ & $\mathrm{R}$ & $\mathrm{R}$ & $\mathrm{S}$ & $\mathrm{R}$ & $\mathrm{R}$ & $\mathrm{R}$ & $\mathrm{S}$ & $\mathrm{R}$ & $\mathrm{S}$ & $\mathrm{S}$ & $\mathrm{S}$ & 1.1.2.7 & I & 16 \\
\hline Cs 86 & $\mathrm{~S}$ & $\mathrm{R}$ & $\mathrm{R}$ & $S$ & $\mathrm{R}$ & $\mathrm{R}$ & $\mathrm{R}$ & $\mathrm{S}$ & $\mathrm{S}$ & $S$ & $\mathrm{R}$ & $\mathrm{R}$ & 1.1 .6 .1 & I & 17 \\
\hline Cs 17 & $\mathrm{~S}$ & $\mathrm{R}$ & $\mathrm{R}$ & $\mathrm{R}$ & $\mathrm{S}$ & $\mathrm{R}$ & $\mathrm{R}$ & $\mathrm{R}$ & $\mathrm{R}$ & $\mathrm{S}$ & $\mathrm{R}$ & $\mathrm{S}$ & 1.2 .0 .5 & I & 18 \\
\hline Cs 84 & $\mathrm{~S}$ & $\mathrm{R}$ & $\mathrm{R}$ & $\mathrm{R}$ & $\mathrm{S}$ & $\mathrm{R}$ & $\mathrm{R}$ & $\mathrm{S}$ & $\mathrm{S}$ & $\mathrm{S}$ & $\mathrm{R}$ & $\mathrm{R}$ & 1.2 .6 .1 & I & 19 \\
\hline Cs 94 & $\mathrm{~S}$ & $\mathrm{R}$ & $\mathrm{R}$ & $\mathrm{R}$ & $\mathrm{S}$ & $\mathrm{R}$ & $\mathrm{R}$ & $\mathrm{S}$ & $\mathrm{S}$ & $\mathrm{S}$ & $\mathrm{R}$ & $\mathrm{R}$ & 1.2 .6 .1 & I & 19 \\
\hline Cs 5 & $\mathrm{~S}$ & $\mathrm{R}$ & $\mathrm{R}$ & $\mathrm{S}$ & $\mathrm{S}$ & $\mathrm{R}$ & $\mathrm{R}$ & $\mathrm{S}$ & $\mathrm{R}$ & $\mathrm{S}$ & $\mathrm{R}$ & $\mathrm{S}$ & 1.3 .2 .5 & I & 20 \\
\hline Cs 80 & $\mathrm{~S}$ & $\mathrm{R}$ & $\mathrm{R}$ & $\mathrm{S}$ & $\mathrm{S}$ & $\mathrm{R}$ & $\mathrm{R}$ & $\mathrm{S}$ & $\mathrm{S}$ & $\mathrm{S}$ & $\mathrm{R}$ & $\mathrm{S}$ & 1.3 .6 .5 & I & 21 \\
\hline Cs 22 & $\mathrm{~S}$ & $\mathrm{R}$ & $\mathrm{R}$ & $\mathrm{S}$ & $\mathrm{S}$ & $\mathrm{R}$ & $\mathrm{R}$ & $\mathrm{S}$ & S & $\mathrm{S}$ & $\mathrm{S}$ & $\mathrm{S}$ & 1.3 .6 .7 & I & 22 \\
\hline Cs 70 & $S$ & $\mathrm{R}$ & $\mathrm{R}$ & $\mathrm{R}$ & $\mathrm{R}$ & $\mathrm{S}$ & $\mathrm{R}$ & $\mathrm{R}$ & $\mathrm{S}$ & $S$ & $\mathrm{R}$ & $\mathrm{R}$ & 1.4.4.1 & I & 23 \\
\hline Cs 20 & $\mathrm{~S}$ & $\mathrm{R}$ & $\mathrm{R}$ & $\mathrm{R}$ & $\mathrm{R}$ & $\mathrm{S}$ & $\mathrm{R}$ & $\mathrm{S}$ & $\mathrm{S}$ & $\mathrm{S}$ & $\mathrm{R}$ & $\mathrm{R}$ & 1.4.6.1 & I & 24 \\
\hline Cs 61 & $\mathrm{~S}$ & $\mathrm{R}$ & $\mathrm{R}$ & $\mathrm{S}$ & $\mathrm{R}$ & $\mathrm{S}$ & $\mathrm{S}$ & $\mathrm{S}$ & $\mathrm{R}$ & $\mathrm{S}$ & S & $\mathrm{S}$ & 1.5 .3 .7 & I & 25 \\
\hline Cs 25 & $\mathrm{~S}$ & $\mathrm{R}$ & $\mathrm{R}$ & $\mathrm{S}$ & $\mathrm{R}$ & $\mathrm{S}$ & $\mathrm{R}$ & $\mathrm{S}$ & $\mathrm{S}$ & $\mathrm{S}$ & $\mathrm{R}$ & $\mathrm{R}$ & 1.5.6.1 & I & 26 \\
\hline Cs 13 & $\mathrm{~S}$ & $\mathrm{R}$ & $\mathrm{R}$ & S & $\mathrm{R}$ & $\mathrm{S}$ & $\mathrm{R}$ & $\mathrm{S}$ & $\mathrm{S}$ & $\mathrm{S}$ & $\mathrm{R}$ & $\mathrm{R}$ & 1.5.6.1 & I & 26 \\
\hline Cs 35 & $\mathrm{~S}$ & $\mathrm{R}$ & $\mathrm{R}$ & $\mathrm{S}$ & $\mathrm{R}$ & $\mathrm{S}$ & $\mathrm{R}$ & $\mathrm{S}$ & $\mathrm{S}$ & $\mathrm{S}$ & $\mathrm{R}$ & $\mathrm{R}$ & 1.5.6.1 & I & 27 \\
\hline Cs 43 & $\mathrm{~S}$ & $\mathrm{R}$ & $\mathrm{R}$ & $\mathrm{S}$ & $\mathrm{R}$ & $\mathrm{S}$ & $\mathrm{R}$ & $\mathrm{S}$ & $\mathrm{S}$ & $\mathrm{S}$ & $\mathrm{R}$ & $\mathrm{R}$ & 1.5 .6 .3 & I & 27 \\
\hline Cs 75 & $\mathrm{~S}$ & $\mathrm{R}$ & $\mathrm{R}$ & $\mathrm{S}$ & $\mathrm{R}$ & $\mathrm{S}$ & $\mathrm{R}$ & $\mathrm{S}$ & $\mathrm{S}$ & $\mathrm{S}$ & $\mathrm{R}$ & $S$ & 1.5 .6 .5 & I & 28 \\
\hline Cs 60 & $\mathrm{~S}$ & $\mathrm{R}$ & $\mathrm{R}$ & $\mathrm{R}$ & $\mathrm{R}$ & $\mathrm{S}$ & $\mathrm{R}$ & $\mathrm{S}$ & $\mathrm{S}$ & $\mathrm{R}$ & $\mathrm{R}$ & $\mathrm{S}$ & 1.4 .6 .4 & I & 29 \\
\hline Cs 91 & $\mathrm{~S}$ & $\mathrm{R}$ & $\mathrm{R}$ & $\mathrm{S}$ & $\mathrm{R}$ & $\mathrm{S}$ & $\mathrm{R}$ & S & $S$ & $\mathrm{~S}$ & $\mathrm{~S}$ & $\mathrm{~S}$ & 1.5 .6 .7 & I & 30 \\
\hline Cs 34 & $\mathrm{~S}$ & $\mathrm{R}$ & $\mathrm{R}$ & $\mathrm{S}$ & $\mathrm{R}$ & $\mathrm{S}$ & $\mathrm{R}$ & $\mathrm{S}$ & $\mathrm{S}$ & $\mathrm{S}$ & $\mathrm{S}$ & $\mathrm{S}$ & 1.5.6.7 & I & 20 \\
\hline Cs 89 & $\mathrm{~S}$ & $\mathrm{R}$ & $\mathrm{R}$ & $\mathrm{R}$ & $\mathrm{S}$ & $\mathrm{S}$ & $\mathrm{R}$ & $\mathrm{S}$ & S & $\mathrm{S}$ & $\mathrm{S}$ & $\mathrm{R}$ & 1.6 .6 .3 & I & 31 \\
\hline Cs 27 & $\mathrm{~S}$ & $\mathrm{R}$ & $\mathrm{R}$ & $\mathrm{R}$ & $\mathrm{S}$ & $\mathrm{S}$ & $\mathrm{R}$ & $S$ & $S$ & $\mathrm{~S}$ & $\mathrm{~S}$ & $\mathrm{R}$ & 1.6 .6 .3 & I & 31 \\
\hline Cs 85 & $\mathrm{~S}$ & $\mathrm{R}$ & $\mathrm{R}$ & $\mathrm{R}$ & $\mathrm{S}$ & $\mathrm{S}$ & $\mathrm{R}$ & $\mathrm{S}$ & $\mathrm{S}$ & $\mathrm{S}$ & $\mathrm{S}$ & $\mathrm{R}$ & 1.6 .6 .3 & I & 31 \\
\hline Cs 50 & S & $\mathrm{R}$ & $\mathrm{R}$ & $\mathrm{S}$ & S & S & $\mathrm{R}$ & $\mathrm{R}$ & $\mathrm{S}$ & $\mathrm{S}$ & $\mathrm{S}$ & $\mathrm{S}$ & 1.7 .4 .7 & I & 32 \\
\hline Cs 69 & $\mathrm{~S}$ & $\mathrm{R}$ & $\mathrm{R}$ & $\mathrm{S}$ & $\mathrm{S}$ & $\mathrm{S}$ & $\mathrm{R}$ & $\mathrm{S}$ & $\mathrm{S}$ & $\mathrm{S}$ & $\mathrm{R}$ & $\mathrm{R}$ & 1.7 .6 .1 & I & 33 \\
\hline Cs 66 & $\mathrm{~S}$ & $\mathrm{R}$ & $\mathrm{R}$ & $\mathrm{S}$ & $\mathrm{S}$ & $\mathrm{S}$ & $\mathrm{R}$ & $\mathrm{S}$ & $\mathrm{S}$ & $\mathrm{S}$ & $\mathrm{R}$ & $\mathrm{R}$ & 1.7 .6 .1 & I & 33 \\
\hline Cs 83 & $\mathrm{~S}$ & $\mathrm{R}$ & $\mathrm{R}$ & $\mathrm{S}$ & $\mathrm{S}$ & $\mathrm{S}$ & $\mathrm{R}$ & $\mathrm{S}$ & $\mathrm{S}$ & $\mathrm{S}$ & $\mathrm{R}$ & $\mathrm{R}$ & 1.7.6.1 & I & 34 \\
\hline Cs 79 & $\mathrm{~S}$ & $\mathrm{R}$ & $\mathrm{R}$ & $\mathrm{S}$ & $\mathrm{S}$ & $\mathrm{S}$ & $\mathrm{R}$ & $\mathrm{S}$ & $\mathrm{S}$ & $\mathrm{S}$ & $\mathrm{R}$ & $\mathrm{R}$ & 1.7 .6 .2 & I & 35 \\
\hline Cs 55 & $\mathrm{~S}$ & $\mathrm{R}$ & $\mathrm{R}$ & $\mathrm{S}$ & $\mathrm{S}$ & $\mathrm{S}$ & $\mathrm{R}$ & $\mathrm{S}$ & $\mathrm{S}$ & $\mathrm{S}$ & $\mathrm{S}$ & $\mathrm{R}$ & 1.7 .6 .3 & I & 36 \\
\hline Cs 57 & $\mathrm{~S}$ & $\mathrm{R}$ & $\mathrm{R}$ & $\mathrm{S}$ & $\mathrm{S}$ & $\mathrm{S}$ & $\mathrm{R}$ & $\mathrm{S}$ & $\mathrm{S}$ & $\mathrm{S}$ & $\mathrm{R}$ & $\mathrm{S}$ & 1.7 .6 .5 & I & 37 \\
\hline Cs 38 & S & $\mathrm{R}$ & $\mathrm{R}$ & $\mathrm{S}$ & $S$ & S & $\mathrm{R}$ & $S$ & S & $S$ & $\mathrm{R}$ & $S$ & 1.7 .6 .5 & I & 37 \\
\hline Cs 19 & $\mathrm{~S}$ & $\mathrm{R}$ & $\mathrm{R}$ & $\mathrm{S}$ & $\mathrm{S}$ & $\mathrm{S}$ & $\mathrm{R}$ & $\mathrm{S}$ & $\mathrm{S}$ & $\mathrm{S}$ & $\mathrm{R}$ & $\mathrm{S}$ & 1.7 .6 .5 & I & 37 \\
\hline Cs 81 & $\mathrm{~S}$ & $\mathrm{R}$ & $\mathrm{R}$ & $\mathrm{S}$ & $\mathrm{S}$ & $\mathrm{S}$ & $\mathrm{R}$ & $\mathrm{S}$ & $\mathrm{S}$ & $\mathrm{S}$ & $\mathrm{S}$ & $\mathrm{S}$ & 1.7 .6 .7 & I & 38 \\
\hline Cs 43 & $\mathrm{~S}$ & $\mathrm{R}$ & $\mathrm{R}$ & $\mathrm{S}$ & $\mathrm{S}$ & $\mathrm{S}$ & $\mathrm{R}$ & $\mathrm{S}$ & $\mathrm{S}$ & $\mathrm{S}$ & $\mathrm{S}$ & $\mathrm{S}$ & 1.7.6.7 & I & 38 \\
\hline Cs 58 & $\mathrm{~S}$ & $\mathrm{R}$ & $\mathrm{R}$ & $\mathrm{S}$ & $\mathrm{S}$ & $\mathrm{S}$ & $\mathrm{R}$ & $\mathrm{S}$ & $\mathrm{S}$ & $\mathrm{S}$ & $\mathrm{S}$ & $\mathrm{S}$ & 1.7 .6 .7 & I & 38 \\
\hline
\end{tabular}

${ }^{a}$ One isolate (Cs 29) from Nepal (Plain) was not included in data analysis due to poor growth. $\mathrm{R}=$ resistant and $\mathrm{S}=$ susceptible.

${ }^{\mathrm{b}}$ Nomenclature code. Virulence of each isolate of $C$. sativus was evaluated on 12 differential wheat lines based the infection response (IR) and was assessed 9 to 10 days after inoculation using a 1-to-9 ordinal disease rating scale (16). The triplet coded nomenclature system as proposed by Limpert and Muller (27) was used to classify virulence of the 95 isolates of $C$. sativus. Nonparametric analysis (39) was performed using IR scores to obtain median disease values for each line-isolate combination as described in Materials and Methods.

${ }^{c}$ Virulence group type: $\mathrm{L}=$ low, $\mathrm{I}=$ intermediate, and $\mathrm{H}=$ high.

d Pathotype was identified according to virulence patterns on differential wheat lines and triplet coded nomenclature system as proposed by Limpert and Muller (27). 
Table 3. (continued from preceding page)

\begin{tabular}{|c|c|c|c|c|c|c|c|c|c|c|c|c|c|c|c|}
\hline Isolate & BL 1473 & Chirya-7 & Chirya- 3 & G 162 & MS-7 & $\begin{array}{l}\text { Ning } \\
8201\end{array}$ & $\begin{array}{l}\text { Ning } \\
8319\end{array}$ & Ocepar-7 & PBW 343 & Sonalika & $\begin{array}{c}\text { SW 89- } \\
5422\end{array}$ & Yangmai-6 & Code $^{b}$ & Group $^{c}$ & Pathotype $^{\mathrm{d}}$ \\
\hline Cs 15 & $\mathrm{~S}$ & $\mathrm{R}$ & $\mathrm{R}$ & $\mathrm{S}$ & $\mathrm{S}$ & $\mathrm{S}$ & $\mathrm{R}$ & $\mathrm{S}$ & $\mathrm{S}$ & $\mathrm{S}$ & $\mathrm{S}$ & $\mathrm{S}$ & 1.7.6.7 & I & 38 \\
\hline Cs 23 & $\mathrm{~S}$ & $\mathrm{R}$ & $\mathrm{R}$ & $\mathrm{S}$ & $\mathrm{S}$ & $\mathrm{S}$ & $\mathrm{R}$ & $\mathrm{S}$ & $\mathrm{S}$ & $\mathrm{S}$ & $\mathrm{S}$ & $\mathrm{S}$ & 1.7.6.7 & I & 38 \\
\hline Cs11 & $\mathrm{S}$ & $\mathrm{R}$ & $\mathrm{R}$ & $\mathrm{S}$ & $\mathrm{S}$ & $\mathrm{S}$ & $\mathrm{R}$ & $\mathrm{S}$ & $\mathrm{S}$ & $\mathrm{S}$ & $\mathrm{S}$ & $\mathrm{S}$ & 1.7.6.7 & I & 38 \\
\hline Cs 72 & $\mathrm{~S}$ & $\mathrm{R}$ & $\mathrm{R}$ & $\mathrm{S}$ & $\mathrm{S}$ & $\mathrm{S}$ & $\mathrm{R}$ & $\mathrm{S}$ & $\mathrm{S}$ & $\mathrm{S}$ & $\mathrm{S}$ & $\mathrm{S}$ & 1.7.6.7 & I & 38 \\
\hline Cs 56 & $\mathrm{R}$ & $\mathrm{S}$ & $\mathrm{R}$ & $\mathrm{R}$ & $\mathrm{R}$ & $\mathrm{S}$ & $\mathrm{R}$ & $\mathrm{S}$ & $\mathrm{S}$ & $\mathrm{S}$ & $\mathrm{R}$ & $\mathrm{R}$ & 2.4.6.1 & I & 39 \\
\hline Cs 18 & $\mathrm{~S}$ & $\mathrm{R}$ & $\mathrm{S}$ & $\mathrm{R}$ & $\mathrm{R}$ & $\mathrm{S}$ & $\mathrm{R}$ & $\mathrm{S}$ & $\mathrm{S}$ & $\mathrm{S}$ & $\mathrm{S}$ & $\mathrm{S}$ & 5.4.6.7 & I & 40 \\
\hline Cs14 & $\mathrm{S}$ & $\mathrm{R}$ & $\mathrm{S}$ & $\mathrm{S}$ & $\mathrm{R}$ & $\mathrm{S}$ & $\mathrm{R}$ & $\mathrm{S}$ & $\mathrm{S}$ & $\mathrm{S}$ & $\mathrm{S}$ & $\mathrm{S}$ & 5.5.6.7 & I & 41 \\
\hline Cs 68 & $\mathrm{~S}$ & $\mathrm{R}$ & $\mathrm{S}$ & $\mathrm{S}$ & $\mathrm{R}$ & $\mathrm{S}$ & $\mathrm{S}$ & $\mathrm{S}$ & $\mathrm{S}$ & $\mathrm{S}$ & $\mathrm{S}$ & $\mathrm{S}$ & 5.5.7.7 & I & 42 \\
\hline Cs 67 & $\mathrm{~S}$ & $\mathrm{R}$ & $S$ & $\mathrm{~S}$ & $\mathrm{~S}$ & $\mathrm{~S}$ & $\mathrm{~S}$ & $\mathrm{~S}$ & $\mathrm{R}$ & $\mathrm{S}$ & $\mathrm{S}$ & $\mathrm{S}$ & 5.7 .3 .7 & I & 43 \\
\hline Cs 54 & $\mathrm{~S}$ & $\mathrm{R}$ & $\mathrm{S}$ & $\mathrm{S}$ & $\mathrm{S}$ & $\mathrm{S}$ & $\mathrm{R}$ & $\mathrm{S}$ & $\mathrm{S}$ & $\mathrm{S}$ & $\mathrm{S}$ & $\mathrm{R}$ & 5.7 .6 .3 & I & 44 \\
\hline Cs 49 & $\mathrm{~S}$ & $\mathrm{R}$ & $\mathrm{S}$ & $\mathrm{S}$ & $\mathrm{S}$ & $\mathrm{S}$ & $\mathrm{R}$ & $\mathrm{S}$ & $\mathrm{S}$ & $\mathrm{S}$ & $\mathrm{S}$ & $\mathrm{S}$ & 5.7.6.7 & I & 45 \\
\hline Cs 71 & $\mathrm{~S}$ & $\mathrm{R}$ & $\mathrm{S}$ & $\mathrm{S}$ & $\mathrm{S}$ & $\mathrm{S}$ & $\mathrm{R}$ & $\mathrm{S}$ & $\mathrm{S}$ & $\mathrm{S}$ & $\mathrm{S}$ & $\mathrm{S}$ & 5.7.6.7 & I & 45 \\
\hline Cs 76 & $\mathrm{~S}$ & $\mathrm{R}$ & $\mathrm{S}$ & $\mathrm{S}$ & $\mathrm{S}$ & $\mathrm{S}$ & $\mathrm{R}$ & $\mathrm{S}$ & $\mathrm{S}$ & $\mathrm{S}$ & $\mathrm{S}$ & $\mathrm{S}$ & 5.7.6.7 & I & 45 \\
\hline Cs 95 & $\mathrm{~S}$ & $\mathrm{R}$ & $\mathrm{S}$ & $\mathrm{S}$ & $\mathrm{S}$ & $\mathrm{S}$ & $\mathrm{R}$ & $\mathrm{S}$ & $\mathrm{S}$ & $\mathrm{S}$ & $\mathrm{S}$ & $\mathrm{S}$ & 5.7.6.7 & I & 45 \\
\hline Cs 77 & $\mathrm{~S}$ & $\mathrm{R}$ & $\mathrm{S}$ & $\mathrm{S}$ & $\mathrm{S}$ & $\mathrm{S}$ & $\mathrm{R}$ & $\mathrm{S}$ & $\mathrm{S}$ & $\mathrm{S}$ & $\mathrm{S}$ & $\mathrm{S}$ & 5.7.6.7 & I & 45 \\
\hline Cs 48 & $\mathrm{~S}$ & $\mathrm{R}$ & $\mathrm{S}$ & $\mathrm{S}$ & $\mathrm{S}$ & $\mathrm{S}$ & $\mathrm{R}$ & $\mathrm{S}$ & $\mathrm{S}$ & $\mathrm{S}$ & $\mathrm{S}$ & $\mathrm{S}$ & 5.7.6.7 & I & 45 \\
\hline Cs 42 & $\mathrm{~S}$ & $\mathrm{R}$ & $\mathrm{S}$ & $\mathrm{S}$ & $\mathrm{S}$ & $\mathrm{S}$ & $\mathrm{R}$ & $\mathrm{S}$ & $\mathrm{S}$ & $\mathrm{S}$ & $\mathrm{S}$ & $\mathrm{S}$ & 5.7.6.7 & I & 45 \\
\hline Cs 39 & $\mathrm{~S}$ & $\mathrm{R}$ & $\mathrm{S}$ & $\mathrm{S}$ & $\mathrm{S}$ & $\mathrm{S}$ & $\mathrm{R}$ & $\mathrm{S}$ & $\mathrm{S}$ & $\mathrm{S}$ & $\mathrm{S}$ & $\mathrm{S}$ & 5.7.6.7 & I & 45 \\
\hline Cs 37 & $\mathrm{~S}$ & $\mathrm{R}$ & $\mathrm{S}$ & $\mathrm{S}$ & $\mathrm{S}$ & $\mathrm{S}$ & $\mathrm{R}$ & $\mathrm{S}$ & $\mathrm{S}$ & $\mathrm{S}$ & $\mathrm{S}$ & $\mathrm{S}$ & 5.7.6.7 & I & 45 \\
\hline Cs 45 & $\mathrm{~S}$ & $\mathrm{R}$ & $\mathrm{S}$ & $\mathrm{S}$ & $\mathrm{S}$ & $\mathrm{S}$ & $\mathrm{R}$ & $\mathrm{S}$ & $\mathrm{S}$ & $\mathrm{S}$ & $\mathrm{S}$ & $\mathrm{S}$ & 5.7.6.7 & I & 45 \\
\hline Cs 44 & $\mathrm{~S}$ & $\mathrm{R}$ & $\mathrm{S}$ & $\mathrm{S}$ & $\mathrm{S}$ & $\mathrm{S}$ & $\mathrm{R}$ & $\mathrm{S}$ & $\mathrm{S}$ & $\mathrm{S}$ & $\mathrm{S}$ & $\mathrm{S}$ & 5.7.6.7 & I & 45 \\
\hline Cs 32 & $\mathrm{~S}$ & $\mathrm{R}$ & $\mathrm{S}$ & $\mathrm{S}$ & $\mathrm{S}$ & $\mathrm{S}$ & $\mathrm{R}$ & $\mathrm{S}$ & $\mathrm{S}$ & $\mathrm{S}$ & $\mathrm{S}$ & $\mathrm{S}$ & 5.7.6.7 & I & 45 \\
\hline Cs 41 & $\mathrm{~S}$ & $\mathrm{R}$ & $\mathrm{S}$ & $\mathrm{S}$ & $\mathrm{S}$ & $\mathrm{S}$ & $\mathrm{S}$ & $\mathrm{S}$ & $\mathrm{S}$ & $\mathrm{S}$ & $\mathrm{S}$ & $\mathrm{S}$ & 5.7 .7 .7 & I & 46 \\
\hline Cs 47 & $\mathrm{~S}$ & $\mathrm{R}$ & $\mathrm{S}$ & $\mathrm{S}$ & $\mathrm{S}$ & $\mathrm{S}$ & $\mathrm{S}$ & $\mathrm{S}$ & $\mathrm{S}$ & $\mathrm{S}$ & $\mathrm{S}$ & $\mathrm{S}$ & 5.7 .7 .7 & I & 46 \\
\hline Cs 31 & $\mathrm{~S}$ & $\mathrm{R}$ & $\mathrm{S}$ & $\mathrm{S}$ & $\mathrm{S}$ & $\mathrm{S}$ & $\mathrm{S}$ & $\mathrm{S}$ & $\mathrm{S}$ & $\mathrm{S}$ & $\mathrm{S}$ & $\mathrm{S}$ & 5.7 .7 .7 & I & 46 \\
\hline Cs 28 & $\mathrm{~S}$ & $\mathrm{R}$ & $\mathrm{S}$ & $\mathrm{S}$ & $\mathrm{S}$ & $\mathrm{S}$ & $\mathrm{S}$ & $\mathrm{S}$ & $\mathrm{S}$ & $\mathrm{S}$ & $\mathrm{S}$ & $\mathrm{S}$ & 5.7 .7 .7 & I & 46 \\
\hline Cs 33 & $\mathrm{~S}$ & $\mathrm{R}$ & $\mathrm{S}$ & $\mathrm{S}$ & $\mathrm{S}$ & $\mathrm{S}$ & $\mathrm{S}$ & $\mathrm{S}$ & $\mathrm{S}$ & $\mathrm{S}$ & $\mathrm{S}$ & $\mathrm{S}$ & 5.7 .7 .7 & I & 46 \\
\hline Cs 24 & $\mathrm{~S}$ & $\mathrm{~S}$ & $\mathrm{~S}$ & $\mathrm{~S}$ & $\mathrm{~S}$ & $\mathrm{~S}$ & $\mathrm{~S}$ & $\mathrm{~S}$ & $\mathrm{~S}$ & $\mathrm{~S}$ & $\mathrm{~S}$ & $\mathrm{~S}$ & 7.7.7.7 & $\mathrm{H}$ & 47 \\
\hline Cs 40 & $\mathrm{~S}$ & $\mathrm{~S}$ & $\mathrm{~S}$ & $\mathrm{~S}$ & $\mathrm{~S}$ & $\mathrm{~S}$ & $\mathrm{~S}$ & $\mathrm{~S}$ & $\mathrm{~S}$ & $\mathrm{~S}$ & $\mathrm{~S}$ & $\mathrm{~S}$ & 7.7.7.7 & $\mathrm{H}$ & 47 \\
\hline Cs 3 & $\mathrm{~S}$ & $\mathrm{~S}$ & $\mathrm{~S}$ & $\mathrm{~S}$ & $\mathrm{~S}$ & $\mathrm{~S}$ & $\mathrm{~S}$ & $\mathrm{~S}$ & $\mathrm{~S}$ & $\mathrm{~S}$ & $\mathrm{~S}$ & $\mathrm{~S}$ & 7.7.7.7 & $\mathrm{H}$ & 47 \\
\hline Cs 46 & $\mathrm{~S}$ & $\mathrm{~S}$ & $\mathrm{~S}$ & $\mathrm{~S}$ & $\mathrm{~S}$ & $\mathrm{~S}$ & $\mathrm{~S}$ & $\mathrm{~S}$ & $\mathrm{~S}$ & $\mathrm{~S}$ & $\mathrm{~S}$ & $\mathrm{~S}$ & 7.7.7.7 & $\mathrm{H}$ & 47 \\
\hline
\end{tabular}

$1.5 \mathrm{mM} \mathrm{MgCl} 2$, and 1 unit of Taq DNA polymerase at $94^{\circ} \mathrm{C}$ for 30 s, $56^{\circ} \mathrm{C}$ for $60 \mathrm{~s}$, and $72^{\circ} \mathrm{C}$ for $60 \mathrm{~s}$ for 20 cycles. The EcoRI primers were labeled with the fluorescent dye IRD 700 (Li-COR Biosciences) and unlabeled $M s e I$ primers with two selective bases were used for selective amplification.

Eight EcoRI and MseI primer combinations (E-AA+M-CA, E$\mathrm{AA}+\mathrm{M}-\mathrm{CC}, \mathrm{E}-\mathrm{AC}+\mathrm{M}-\mathrm{CA}, \mathrm{E}-\mathrm{AC}+\mathrm{M}-\mathrm{CC}, \mathrm{E}-\mathrm{AC}+\mathrm{M}-\mathrm{CT}, \mathrm{E}-\mathrm{AG}+\mathrm{M}-$ $\mathrm{CA}$, E-AG+M-CG, and $\mathrm{E}-\mathrm{AT}+\mathrm{M}-\mathrm{CT}$ ) revealed many polymorphisms in our preliminary as well as previous study (56). Therefore, these eight primer combinations were used in AFLP analysis (Table 4). Selective amplifications were done using the PCR conditions as described by Vos et al. (50). The selective amplified reactions were mixed with $6 \mu \mathrm{l}$ of formamide loading dye and later denatured at $95^{\circ} \mathrm{C}$ for $5 \mathrm{~min}$. Each PCR was run twice to determine variability between runs. The amplified products were separated on a LI-COR Global edition IR $^{2}$ System (Li-COR Biosciences) in $6 \%$ polyacrylamide gels within $1 \times$ TBE buffer (10.8 $\mathrm{g}$ of Tris base, 5.5 $\mathrm{g}$ of boric acid, $2 \mathrm{ml}$ of $0.5 \mathrm{M}$ EDTA, and $1,000 \mathrm{ml}$ of sterile distilled water) as described previously (56). The polyacrylamide gel solution contained $20 \mathrm{ml}$ of Rapid Gel-XL 6\% liquid acrylamide (USB Corporation), $150 \mu \mathrm{l}$ of $10 \%$ fresh ammonium persulfate, and $15 \mu \mathrm{l}$ of tetramethylethylenediamine. The gel electrophoresis was run at $2,000 \mathrm{~V}$ with $40 \mathrm{~mA}$ of current and $50 \mathrm{~W}$ of power at $45^{\circ} \mathrm{C}$ temperature for $3 \mathrm{~h}$ with a Global $\mathrm{IR}^{2}$ System (Li-COR Biosciences). Band sizes of 60 to $700 \mathrm{bp}$ that were reproducible were scored manually with reference to a 50-bp ladder (Li-COR Biosciences). The DNA fragments were scored manually in a binary system, where presence of a band was coded as 1 and absence as 0 . From the dissimilarity matrix, a dendrogram was obtained using the unweighted pair group mean analysis (UPGMA) with the SIMINT program in NTSYS-pc software (version 2.1; Exeter Software). To determine the robustness of the dendrogram, the presence or absence of data were resampled by replacement with 1,000 bootstrap replicates.

AFLP data analysis. The data analyses were performed using repeatable and polymorphic amplified bands. Different fragment
Table 4. Number of polymorphic amplified fragment length polymorphism bands generated by eight primer combinations in 96 isolates of Cochliobolus sativus from Nepal and North Dakota

\begin{tabular}{lcc}
\hline Primer combinations & Total bands & Polymorphic bands (\%) \\
\hline EcoRI-AA/MseI-CA & 39 & 26 \\
EcoRI-AA/MseI-CC & 35 & 31 \\
EcoRI-AC/MseI-CA & 27 & 43 \\
EcoRI-AC/MseI-CC & 28 & 89 \\
EcoRI-AC/MseI-CT & 38 & 34 \\
EcoRI-AG/MseI-CA & 38 & 82 \\
EcoRI-AG/MseI-CG & 36 & 27 \\
EcoRI-AT/MseI-CT & 41 & 54 \\
Total & 272 & $\ldots$ \\
\hline
\end{tabular}

sizes of each AFLP marker were considered different alleles. Different allele sizes generated by each of the AFLP markers were recorded and entered in the GenAlex 6 format.

Population structure analysis. The affiliation of individual $C$. sativus isolates from sampling groups to specific genetic clusters and the possibility of admixture were tested using the software program STRUCTURE v.2.3.2 (35). STRUCTURE v.2.3.2 was run for Nepal (Hill and Plain areas) and North Dakota (Central and Northern areas) populations separately. Repeated gene flow might cause lack of significant population differentiation between sampling groups. For each number of clusters $K(K=1$ through 10$)$, defined as subpopulation, 10 runs were performed with a burn-in period of 10,000 iterations used for each run, and using 100,000 iterations in Monte Carlo Markov chain replications for the analysis. The number of subpopulations between 1 and 10 was analyzed for a total of five replicates using an admixture model. Further, an ad hoc statistic $\Delta K$ was used to confirm the exact number of subpopulation $(K)$ using Structure Harvester software (v0.6.5). Here, the $\Delta K$ is solely based on the rate of change in log probability of data between its successive $K$ values (13).

Genetic diversity analysis. Alleles generated from all loci were compiled to construct multilocus haplotypes (MLHTs). Isolates 
with the same MLHT were considered clones. Clone-corrected data were used for all analyses. Estimation of total number of alleles, gene diversity $(H)$, genetic distance $(D)$, and Nei's unbiased genetic identity $(I)$ were done using POPGENE version 1.32 (54). Genetic diversity in the total population $\left(H_{T}\right)$ and within the geographic population $\left(H_{S}\right)$ were estimated using Nei's unbiased gene diversity. Pairwise measures of population differentiation between C. sativus populations from the Central and Northern areas of North Dakota and between populations from the Hill and Plain areas of Nepal were done separately using a corrected standardized fixation index (i.e., $G^{\prime \prime}$ ST) using GenoDive 2.0 beta version software $(20,31)$.

Linkage disequilibrium analysis. Multilocus linkage disequilibrium (LD) was assessed using the index of association $\left(\bar{r}_{d}\right)$. The $\bar{r}_{d}$ is an alternative measure of index of association and is less sensitive to number of loci when compared with $I_{\mathrm{A}}$. The $\bar{r}_{d}$ varies from 0 (random association of allele at different loci) to 1 (maximum possible LD among loci). The significance of $\bar{r}_{d}$ was tested by randomization $(1,000$ times) procedures by comparing the observed value of $\bar{r}_{d}$ with that expected under the null hypothesis of complete panmixis (2).The $\bar{r}_{d}$ values were estimated for the Hill and Plain areas (Nepal) and for the Central and Northern areas (North Dakota) separately with MULTILOCUS program version $1.3(2)$.

Analysis of molecular variance. Analysis of molecular variance (AMOVA) was used to partition genetic variation within and among populations for detecting population differences (14). Genetic variation was partitioned within and among populations from Central and Northern areas of North Dakota and within and among populations from Hill and Plain areas of Nepal using the Arlequin 3.1 software $(14,15)$. Variance components and $F_{S T}$ values were tested by 1,023 permutations of haplotypes between $C$. sativus populations to determine level of significance at $P \leq 0.001$ (14).

\section{Results}

Virulence analysis. Homogeneity tests performed using Bartlett's $\chi^{2}$ indicated that the variance in the data sets was homogeneous $\left(\chi^{2}=2.98, P=0.19\right)$. Similarly, analysis of variance of the nonparametric data showed that the main effects of wheat differential lines $(P<0.001)$, isolates $(P<0.001)$, and interactions be-

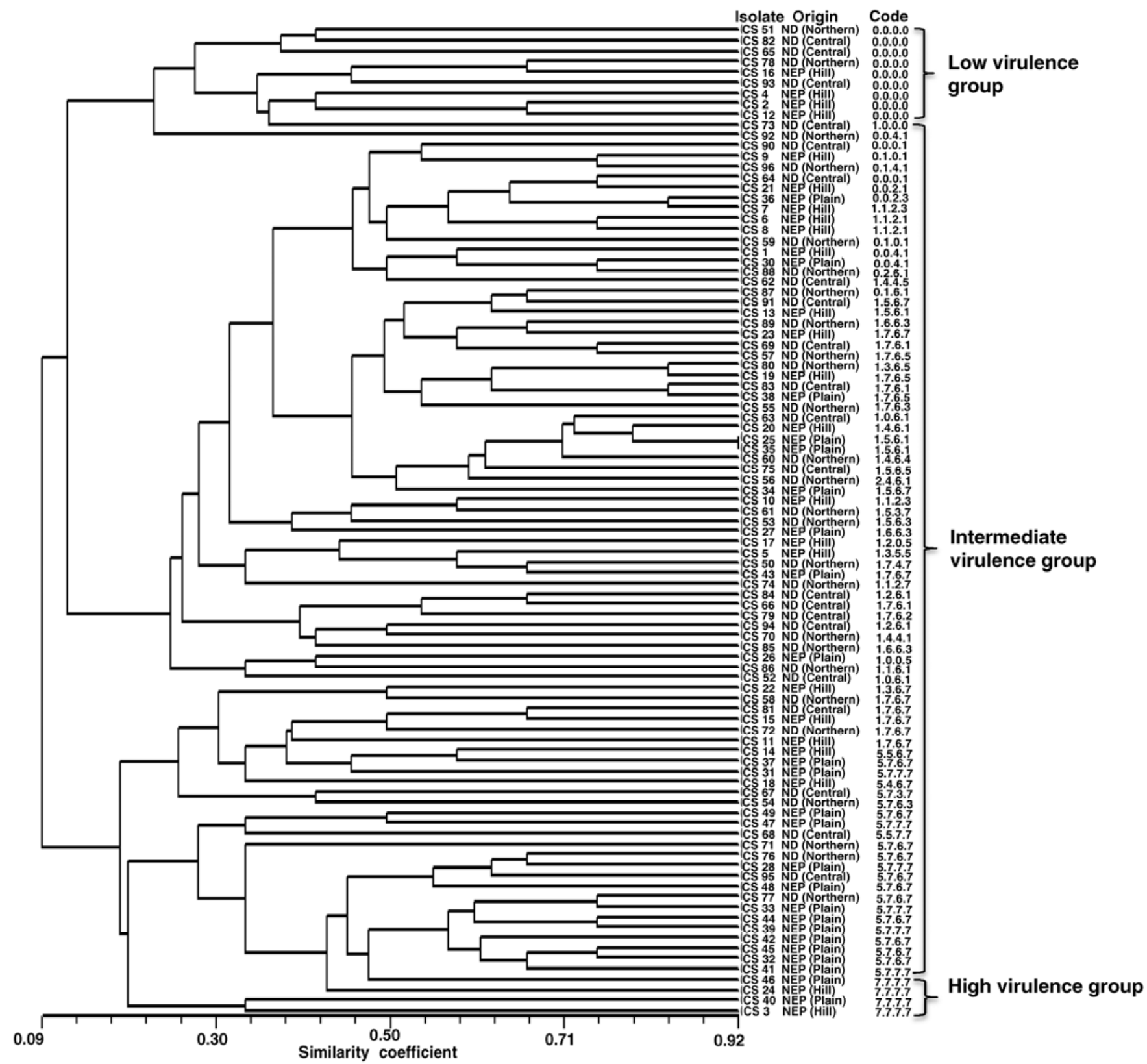

Fig. 1. Dendrogram based on the similarity of the median disease scores of 95 isolates of Cochliobolus sativus on 12 differential wheat lines. Cluster analysis was based on unweighted pair group method using arithmetic averages. Pathotypes were designated according to the coded triplet nomenclature system. 
tween wheat lines and isolates were significant $(P<0.001)$ (data not shown).

All isolates of $C$. sativus produced minute necrotic lesions with no or only slight chlorotic halos to large necrotic lesions surrounded by distinct yellow halos (typical spot blotch symptoms) on 12 differential wheat lines tested. On the basis of hierarchical cluster analysis, the 95 isolates were grouped into 47 pathotypes (Table 3) and were classified into three clusters (Fig. 1). The first cluster had low virulence and contained nine isolates. The second cluster had intermediate virulence isolates and consisted of 82 isolates. The third cluster had four isolates with high virulence on all of the 12 wheat differential lines tested (Fig. 1). No relationship was observed between the virulence group and country of origin (Fig. $1)$. Nine of the isolates from pathotype 1 produced small round or oblong necrotic lesions $\left(<2 \mathrm{~mm}^{2}\right)$ with or without restricted chlorotic margins on susceptible wheat line Sonalika (Table 3 ). The majority of isolates ( 82 isolates from pathotype 2 through pathotype 46) were classified as having intermediate virulence based on their ability to cause susceptible reactions on two susceptible wheat lines and on three to eight resistant wheat lines used in this study. The remaining four isolates from pathotype 47 were classi- fied as highly virulent based on their ability to cause susceptible reactions in all of the resistant wheat lines used in this study. Pathotypes 45, 1, and 38 had the highest number of isolates consisting of 12, 9, and 7 isolates in each group, respectively (Table 3 ). The 30 randomly selected $C$. sativus isolates from Nepal and North Dakota produced minute necrotic lesions similar to those of resistant reactions on barley differential lines Bowman, NDB112, and ND5883. Based on infection response (IR) on the three barley differential lines and the triplet virulent group (i.e., pathotypes 0) (Supplementary Table S1), even the highly virulent isolate CS 46 was unable to cause susceptible reactions on the three differential barley lines. Thus, the disease reactions or scores of wheat $C$. sativus isolates on these barley differential lines were not included in our analysis. Of the 12 differential wheat lines or cultivars tested, Chirya 7 exhibited resistance to 91 of 95 isolates tested while Sonalika was the most susceptible differential host, being susceptible to 84 of 95 isolates. Other differential cultivars were resistant to 22 to 84 isolates.

Sequence analysis of ITS and gpd regions. The ITS (GenBank accession JX145649) and gpd (JX145648) sequences of the 96 C. sativus isolates were identical. Moreover, the gpd sequences from

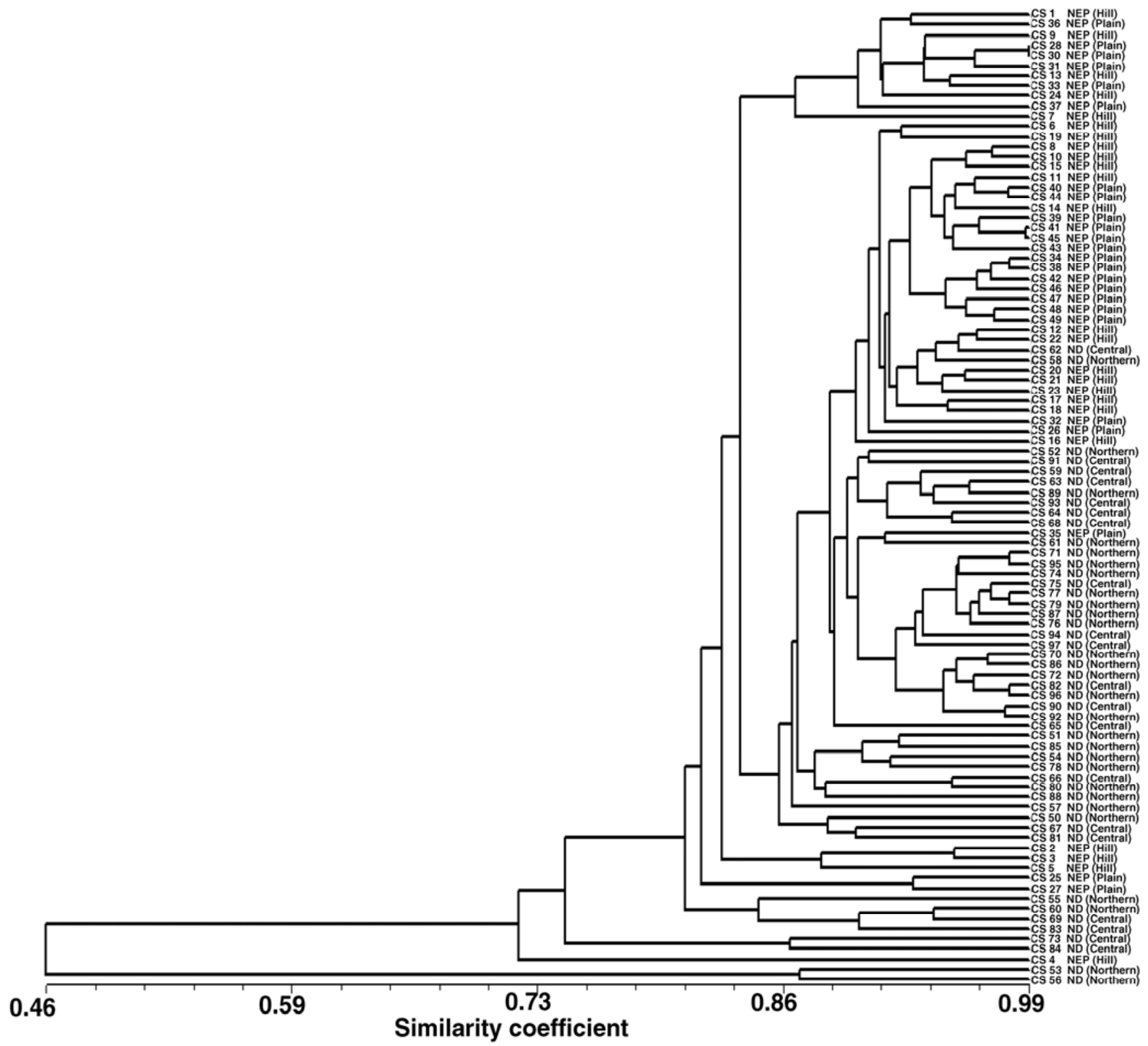

Fig. 2. Estimates of genetic similarity among Cochliobolus sativus isolates collected from the Hill and Plain areas in Nepal and from the Central and Northern areas of North Dakota based on amplified fragment length polymorphisms. Bootstrap values (1,000 replicates) for branch nodes ranged from 10 to $100 \%$. 
C. sativus had 96 to $97 \%$ maximum identity and the ITS sequences had maximum sequence identity from 98 to $99 \%$ from those of the reference isolates of $C$. carbonum, $C$. victoriae, $C$. heterostrophus, and C. miyabeanus (data not shown).

AFLP data analysis. DNA polymorphisms between 60 to 700 bp allowed 108 polymorphic bands from eight AFLP primer combinations across 96 C. sativus isolates to be scored and used for data analysis. On average, 34 bands were scored per primer combination. On average, $48 \%$ polymorphic bands were observed for each primer combination. Two AFLP primer combinations (EcoRI-AC/MseI-CC and EcoRI-AG/MseI-CA) were highly polymorphic for $C$. sativus (Table 4). The dendrogram generated from combined AFLP data from eight primer pair sets revealed that the 96 isolates clustered as two distinct groups using UPGMA with the genetic distance coefficient (Fig. 2). The two isolates from North Dakota (Northern) clustered in one group and all isolates from Nepal (Hill and Plain areas) and North Dakota (Central and Northern areas) clustered together in another group. The isolates from same pathotype and geographic origins were unable to be grouped into the same cluster.

Population structure analysis. The value of $\log \operatorname{Pr}(X / K)$ (i.e., the $\log$ likelihood of the model given that $K$ clusters are present in the data) was significant for $\mathrm{K} 1$ versus $\mathrm{K} 2$ and revealed a single population for the Central and Northern populations from North Dakota and for the Hill and Plain populations from Nepal, respectively (Fig. 3A and B).

Genetic diversity analysis. Gene diversity analysis showed that the $C$. sativus populations had 108 alleles in the entire population. All of the isolates were of distinct haplotypes (Table 5). Gene diversity $(H)$ values for Hill and Plain populations from Nepal were 0.10 and 0.08 , respectively, whereas $H$ values for Central and Northern populations from North Dakota were 0.11 and 0.16 , respectively (Table 5).

LD analysis. Based on the multilocus LD statistic $\left(\bar{r}_{d}\right)$ the null hypothesis of random recombination across loci was rejected for Central, Northern, Hill, and Plain populations from North Dakota and Nepal ( $\bar{r}_{d}=0.066$ to $0.292, P>0.001$; Table 5), suggesting that all MLHTs resulted from asexual reproduction or parasexual recombination. Pairwise comparison of Nei's unbiased genetic identity $(I)$ and genetic distance between the Central and Northern populations from North Dakota were 0.99 and 0.03, respectively.
Similarly, pairwise comparison of Nei's unbiased genetic identity (I) and genetic distance between the Hill and Plain populations from Nepal were 0.98 and 0.01 , respectively. The $G_{\text {ST }}^{\prime \prime}$, which is a corrected standardized fixation index that is calculated as an independent measure of differentiation between populations, was 0.02 between Central and Northern populations from North Dakota and 0.05 between Hill and Plain populations from Nepal.

AMOVA. AMOVA revealed low genetic variation (4\%) among Central and Northern populations whereas greater than $96 \%$ of the genetic variation was found within Central and Northern populations (Table 6). Similarly, low genetic variation (7\%) also was observed among Hill and Plain populations from Nepal, whereas greater than $93 \%$ of the genetic variation was found within Hill and Plain populations. Individuals within Central and Northern populations and within Hill and Plain populations were statistically significant at $P<0.001$ (Table 6).

\section{Discussion}

Virulence analysis of $C$. sativus on differential wheat lines and population genetic analysis using AFLP markers demonstrated that this pathogen is highly pathogenic and genetically diverse in Nepal and North Dakota. To our knowledge, this is the first study to determine virulence and molecular diversity of $C$. sativus populations from wheat foliar infections.

Valjavec-Gratian and Steffenson (48) were the first to develop the pathotype system for $C$. sativus using three barley differential lines (Bowman, NDB112, and ND5883) and using the coded triplet nomenclature system. They reported pathotypes 0,1 , and 2 for $C$. sativus isolated from barley leaves from North Dakota. Since then, the virulence spectra of $C$. sativus from barley have been analyzed in Australia, Canada, and the United States using the coded triplet nomenclature $(17,18,23,48,56)$. Ghazvini and Tekauz (17) used 12 differential barley lines and identified eight virulence groups from 127 C. sativus isolates collected from barley in Canada. In another study, Arabi and Jawhar (4) found virulence of C. sativus from barley in Syria to be highly variable on barley lines possessing different levels of resistance. A study conducted in Australia identified 11 pathotypes among the $31 \mathrm{C}$. sativus isolates collected from barley and wheat, using 12 differential barley lines (23). All of the previous studies have used differential barley lines and the coded triplet nomenclature system to identify pathotypes of $C$. sativus

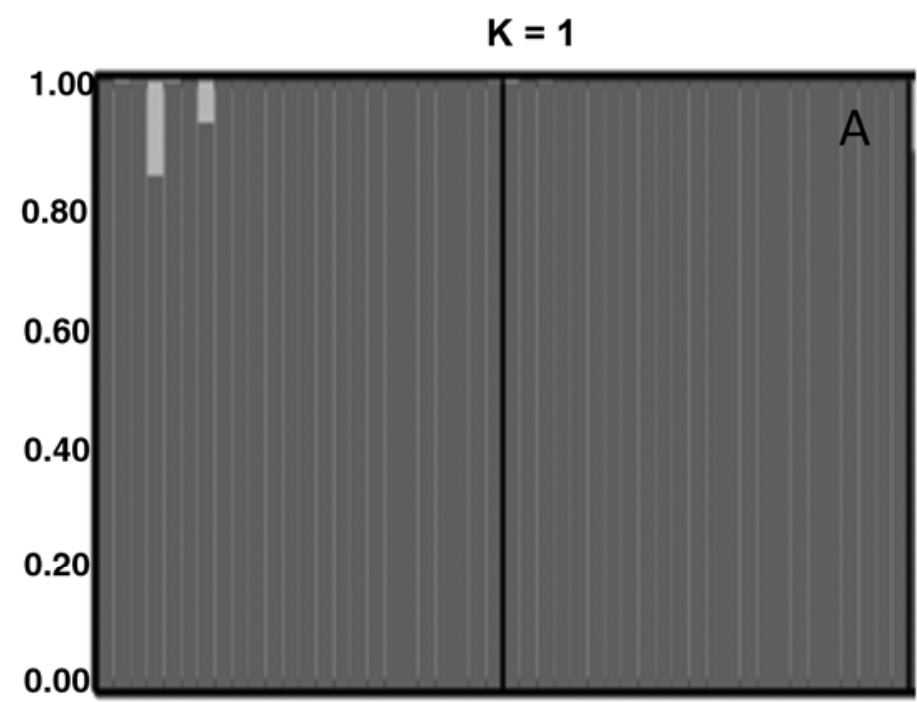

Hill
Plain

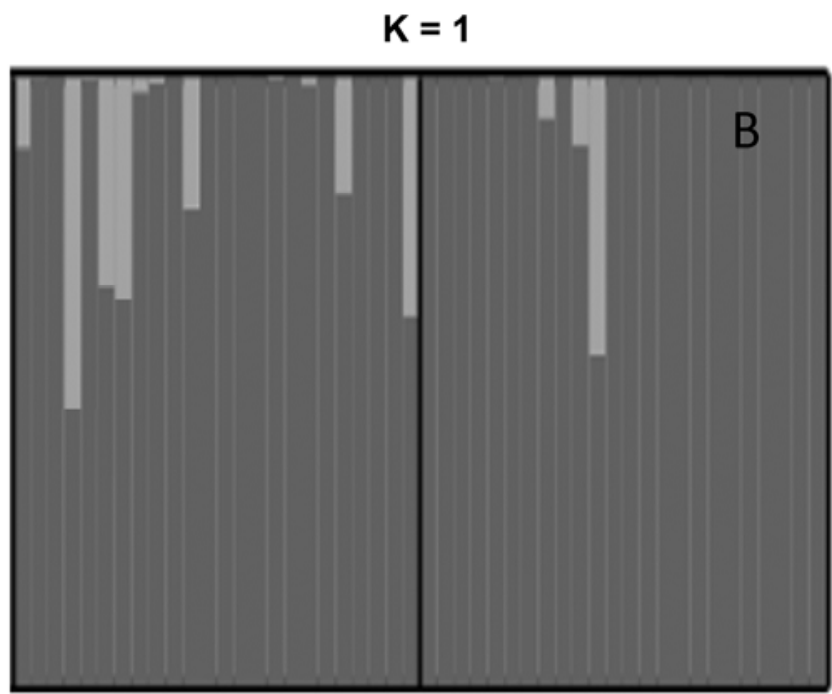

Central

Northern

North Dakota (United States)

Fig. 3. Affiliation of individual genotypes of Cochliobolus sativus as assessed using 108 polymorphic amplified fragment length polymorphism loci measured in Structure ver. 2.33 (35). A, Populations from the Hill and Plain areas of Nepal were determined to be $K=1$ and $B$, populations from the Northern and Central areas of North Dakota were also determined to be $K=1$. 
$(4,17,23,48)$. In this study, we found that $C$. sativus isolates from wheat can only induce minute necrotic lesions, similar to resistant reactions, on the three previously identified barley differential lines Bowman, NDB112, and ND5883. On the basis of IR on barley differential lines, all 30 randomly selected $C$. sativus isolates were grouped into pathotype 0 and were the least virulent pathotype. In contrast, these same isolates showed differential responses on the differential wheat lines tested and could be grouped into low- to high-virulence pathotypes. A similar result was reported by Knight et al. (23), who found that all of the C. sativus isolates from wheat roots were the least virulent (pathotype 0.0.0.0) on 12 differential barley lines. They further reported that the $C$. sativus population from wheat was clearly differentiated from the $C$. sativus population from barley on the basis of the AFLP marker system (23). Similarly, Conner and Atkinson (8) also found that $C$. sativus from wheat roots was less virulent on barley roots and vice versa. Our finding that the isolates from wheat were less virulent on barley differential lines indicates that there is the potential for host specificity (23). Thus, there is a need for differential wheat lines which can clearly define the virulence pattern of $C$. sativus isolated from wheat. We selected 12 wheat cultivars or lines based on their differential responses to $C$. sativus isolates in previous studies $(24,25,30,33,40-44)$. We assume that this could be the first step for identifying such differential wheat lines to determine virulence in $C$. sativus.

All 95 isolates of $C$. sativus were classified into low, intermediate, and high virulence. The majority $(86 \%)$ of isolates had intermediate virulence. The findings of intermediate virulence of wheat-C. sativus interactions suggest that the resistance in the host to the pathogen may be complex and governed by minor genes. Alternatively, there could be the possibility of differences in the virulence factors in this fungus. Kumar et al. $(24,25)$ reported several major and minor resistance loci in some resistant wheat lines. Identification and characterization of necrotropic effectors in this fungus need to be investigated.

Table 5. Genetic diversity of Cochliobolus sativus populations from Hill and Plain areas in Nepal, and Central and Northern areas in North Dakota

\begin{tabular}{lcccc}
\hline Population & Sample size $^{\mathbf{a}}$ & Clonal fraction $^{\mathbf{b}}$ & $\boldsymbol{H}^{\mathbf{c}}$ & $\overline{\boldsymbol{r}}_{\boldsymbol{d}}^{\mathbf{d}}$ \\
\hline Nepal & & & & \\
Hill & 24 & 0.00 & 0.10 & $0.075^{* *}$ \\
Plain & 24 & 0.00 & 0.08 & $0.066^{* *}$ \\
$\quad$ Total & 48 & 0.00 & 0.16 & $\ldots$ \\
North Dakota & & & & \\
$\quad$ Central & 24 & 0.00 & 0.11 & $0.082^{* *}$ \\
Northern & 24 & 0.00 & 0.16 & $0.292^{* *}$ \\
Total & 48 & 0.00 & 0.14 & $\ldots$ \\
\hline
\end{tabular}

${ }^{a}$ Clone-corrected sample size.

${ }^{\mathrm{b}}$ Estimated as $1-[$ (number of different genotypes)/(total number of isolates)] (19).

${ }^{\mathrm{c}} H=$ gene diversity (POPGENE software; 54) within populations was calculated from clone-corrected data.

d Standardized index of association ( $\bar{r}_{d}$ ) statistic was estimated for each population (2) from clone-corrected data. Significance of $\bar{r}_{d}$ was tested with 1,000 randomizations of the data by comparing the observed value with that expected under the null hypothesis of $\bar{r}_{d}=0$. The null hypothesis of multilocus linkage equilibrium was rejected if $P \leq 0.01$ or $0.05 ; * *=$ significant at $P \leq 0.01$ level.
The presence of highly virulent isolates of $C$. sativus in Nepal and North Dakota has raised a serious question regarding the effectiveness of "durable" resistance to $C$. sativus in these regions. The ability of a given population of $C$. sativus to quickly overcome the resistance presently deployed in the field is evident from this study. This study showed that there is immense need of resistance sources that provide better resistance than is currently available in commercial cultivars. Similarly, higher overall disease severity caused by highly virulent isolates from Nepal suggests that the aggressiveness of $C$. sativus population is shifting toward more aggressive types. Several wheat lines have been tested for resistance to $C$. sativus across multi-environments in South Asia (40-44). Most of these resistant lines originated from Brazil, China, and Zambia (11). A few resistant lines have also been derived from crosses with Aegilops tauschii and tetraploid wheat (42). Most of the lines tested across multiple locations have not shown stable or consistent resistance to $C$. sativus; however, three of the wheat genotypes (SW 89-5422, Yangmai-6, and Ning 8201) were reported to have stable spot blotch resistance across 20 environments in Bangladesh, India, and Nepal (41). In another study, Milan Shanghai 7 exhibited a high level of disease resistance and grain yield across 12 different sites in Bangladesh, India, and Nepal (40). The majority of the resistant lines used in this study had shown some level of resistance to $C$. sativus in the field. However, none of the wheat differential lines were completely resistant to the isolates tested. It is possible that some of these resistant wheat lines may have the genes responsible for resistance to $C$. sativus at the adult plant stage and that the gene might remain ineffective at the seedling stage. Interestingly, we found $27 \%$ (26 of 95 isolates) of the isolates able to break down the resistance in 10 resistant wheat lines. We were able to detect a broad spectrum of $C$. sativus pathogenicity in this study. Therefore, the virulence groups which were able to cause disease in all of the tested wheat differential lines would be the best isolates for screening wheat germplasm to identify additional sources of resistance to spot blotch in wheat.

C. sativus has a high degree of morphological and phenotypic variation $(4,17,23,46,48)$; however, little is known about the genetic variation in this pathogen (10). A high level of intraspecific genetic variability in $C$. sativus from Brazil and India has been reported using a random amplified polymorphic DNA (RAPD) marker $(3,9)$. Baturo et al. (5) found low intraspecies differentiation in $C$. sativus populations collected over 15 years in different regions of Brazil using the same RAPD marker system. The present study provides insights into the genetic structure of $C$. sativus populations from two distinct geographic regions where different wheat genotypes are grown. The molecular diversity for both North Dakota (Northern and Central) and Nepal (Hill and Plain) was high, with 96 putative clones identified from 96 genotyped isolates. The genetic variations detected within individual isolates of $C$. sativus from North Dakota and Nepal populations were high (96 and $93 \%$, respectively). In contrast, the genetic variation between Central and Northern populations from North Dakota was $4 \%$ and between Hill and Plain populations from Nepal was 7\%, and they were also significant $(P<0.001)$.

Svobodova et al. (45) had shown a possible population differentiation among $C$. sativus populations collected from different regions of the Czech Republic using the AFLP marker system.

Table 6. Analysis of molecular variance (AMOVA) testing for genetic differentiation between Hill and Plain populations of Cochliobolus sativus from Nepal and Central and Northern populations of $C$. sativus from North Dakota, United States

\begin{tabular}{lcccc}
\hline AMOVA $^{\mathbf{a}}$ & df & Variation (\%) & $\boldsymbol{F}_{\boldsymbol{S T}}^{\mathbf{b}}$ & \\
\hline Nepal & & & $\boldsymbol{P}$ \\
Among Hill versus Plain populations & 1 & 7 & $\ldots$ & $\ldots$ \\
Within Hill versus Plain populations & 46 & 93 & 0.072 & $\ldots .000$ \\
North Dakota & 1 & 4 & $\ldots$ & $\ldots$ \\
Among Central versus Northern populations & 46 & 96 & 0.019 & 0.000 \\
Within Central versus Northern populations & & & \\
\hline
\end{tabular}

a Genetic variation was grouped among Hill and Plain populations from Nepal and among Central and Northern populations from North Dakota.

${ }^{\mathrm{b}} F_{S T}=$ fixation index and the significance was determined by 1023 random permutations. Clone-corrected data were used for AMOVA. $P=0.001$. 
Similarly, Ruckstuhl (37) found population differentiation among South Asia, Mexico, and Bolivia C. sativus populations using RAPD markers. However, the result from this study contradicted the previous studies $(37,45)$ because we did not find any significant population differentiation between North Dakota and Nepalese populations of $C$. sativus. Our results indicate that $C$. sativus populations from North Dakota (i.e., Northern versus Central) have very low population differentiation $\left(G_{\mathrm{ST}}^{\prime \prime}=0.02\right)$. Similarly, $C$. sativus populations from Nepal (i.e., Hill versus Plain) also have low population differentiation $\left(G_{\text {ST }}^{\prime \prime}=0.05\right)$, and this result was also supported by Structure analysis. Thus, no evidence was found for population differentiation and structuring between $C$. sativus populations from North Dakota and Nepal. Most of the wheat planted in the Northern and Central areas in North Dakota are spring wheat habit and most wheat growers have a common seed source. Similarly, most of the wheat cultivars grown in Plain areas of Nepal are also grown in Hill areas. Because C. sativus is a seedborne pathogen (30), we hypothesize that migration of this fungus could have occurred from infected seed at the time of germplasm exchange between Northern and Central areas in North Dakota and, similarly, between Hill and Plain areas in Nepal. This information is useful for wheat breeders to deploy resistances in breeding programs in Nepal and North Dakota.

Our results showed significant $(P>0.001)$ LD for the Northern and Central populations in North Dakota and the Hill and Plain populations in Nepal. This suggests that sexual reproduction may play a less important role in disease epidemiology of this fungus. Our finding is in agreement with the previous study (45), which reported that recombination has a minor impact on the genetic structure of $C$. sativus populations. This is also true because the perfect stage of this fungus has been rarely observed in the field, and it has been reported only once from the field of Zambia (36). Svobodova et al. (45) observed significant LD in populations of $C$. sativus from Czech Republic, and our result was consistent with this previous study. The detection of significant $\mathrm{LD}$ values in $C$. sativus populations in North Dakota and Nepal is likely due to selection, migration of individuals or admixture of populations, and asexual reproduction.

The fungus reproducing asexually should result in high clonal populations and low genotypic diversity. Zhong and Steffenson (56) reported moderate allelic diversity in $C$. sativus populations from barley in North Dakota. Because the sexual phase of the life cycle of this fungus has not been found in the field, there should be other possible mechanisms for moderate to high genetic as well as pathogenic diversity detected in $C$. sativus. Some of the possible genetic mechanisms could be parasexual recombination (56), or translocations, chromosome deletions, and duplications as previously mentioned by Zhong and Steffenson (54).

In summary, this study demonstrated moderate to high pathogenic and genetic diversity of $C$. sativus populations collected from wheat in Nepal and North Dakota. The C. sativus isolates were classified into low, intermediate, and high virulence, and 47 distinct pathotypes were detected based on wheat cultivars or lines possessing different levels of resistance. Significant interactions between wheat differential lines and fungal isolates, and moderate population differentiation of $C$. sativus, suggest that available single genes for resistance to $C$. sativus are likely ineffective in diverse geographic areas. These virulent isolates of $C$. sativus identified in this study may overcome most of the resistant wheat genotypes that are commonly used in the current wheat breeding programs. There is immense need of wheat cultivars that have durable resistance to this fungus. To effectively manage this disease, wheat breeding programs should be initiated to pyramid different resistance genes in adapted wheat cultivars. In addition, identification of new sources of resistance to these virulent isolates of $C$. sativus from Nepal and North Dakota is warranted.

\section{Acknowledgments}

This research was supported by the North Dakota Wheat Commission and State Board of Agricultural Research and Education, North Dakota. B. N. Mahto was supported by the Council for International Exchange of Scholars; Institute of International Education; the U.S. Fulbright Core Program, Washington, DC and the Fulbright Commission, Nepal; and the Nepal Agricultural Research Council, Nepal. We thank M. Bonman for reviewing the manuscript; and S. Ali, J. Hansen, and N. Banjara for their assistance.

\section{Literature Cited}

1. Adhikari, T. B., Hansen, J. M., Gurung, S., and Bonman, J. M. 2011 Identification of new sources of resistance in winter wheat to multiple strains of Xanthomonas translucens pv. undulosa. Plant Dis. 95:582-588.

2. Agapow, P. M., and Burt, A. 2001. Indices of multilocus linkage disequilibrium. Mol. Ecol. Notes 1:101-102.

3. Aggarwal, R., Singh, V. B., Gurjar, M. S., Gupta, S., and Srinivas, P. 2009. Intraspecific variations in Indian isolates of Bipolaris sorokiniana infecting wheat based on morphological, pathogenic and molecular characters. Indian Phytopathol. 62:449-460.

4. Arabi, M. I. E., and Jawhar, M. 2002. Virulence spectrum to barley (Hordeum vulgare L.) In some isolates of Cochliobolus sativus from Syria. J. Plant Pathol. 84:35-39.

5. Baturo, A., Mehta, Y. R., and Sadowski, Cz. K. 2004. Identification of genetic variability in Bipolaris sorokiniana isolates from wheat in Brazil Summa Phytopathol. 30:470-474.

6. Berbee, M. L., Pirseyedi, M., and Hubbard, S. 1999. Cochliobolus phylogenetics and the origin of known, highly virulent pathogens, inferred from ITS and glyceraldehyde-3-phosphate dehydrogenase gene sequences. Mycologia 91:964-977.

7. Bouajila, A., Zoghlami, N., Ahmed, M. A., Baum, M., Ghorbel, A., and Nazari, K. 2012. Pathogenicity spectra and screening for resistance in barley against Tunisian Pyrenophora teres f. teres. Plant Dis. 96:1569-1575.

8. Conner, R. L., and Atkinson, T. G. 1989. Influence of continuous cropping on severity of common root rot in wheat and barley. Can. J. Plant Pathol. 11:127-132.

9. de Oliviera, A. M. R., Matsumura, A. T. S., Prestes, A. M., and Van der Sand, S. T. 2002. Intraspecific variability of Bipolaris sorokinina isolates determined by random amplified polymorphic DNA (RAPD). Gen. Mol. Res. 4:350-358

10. Dubin, H. J., and Rajaram, S. 1996. Breeding disease-resistant wheats for tropical highlands and lowlands. Annu. Rev. Phytopathol. 34:503-526.

11. Duveiller, E. 2004. Controlling foliar blights of wheat in the rice-wheat systems of Asia. Plant Dis. 88:552-556.

12. Duveiller, E., Kandel, Y. R., Sharma, R. C., and Shrestha, S. M. 2005 Epidemiology of foliar blights (spot blotch and tan spot) of wheat in the plains bordering the Himalayas. Phytopathology 95:248-256.

13. Evanno, G., Regnaut, S., and Goudet, J. 2005. Detecting the number of clusters of individuals using the software Structure: a simulation study. Mol. Ecol. 14:2611-2620.

14. Excoffier, L., Laval, G., and Schneider, S. 2005. Arlequin (version 3.0): an integrated software package for population genetics data analysis. Evol. Bioinf. 1:47-50.

15. Excoffier, L., Smouse, P. E., and Quattro, J. M. 1992. Analysis of molecular variance inferred from metric distances among DNA haplotypes: application to human mitochondrial DNA restriction data. Genetics 131:479-491.

16. Fetch, T. C., and Steffenson, B. J. 1999. Rating scales for assessing infection responses of barley infected with Cochliobolus sativus. Plant Dis. 83:213-217.

17. Ghazvini, H., and Tekauz, A. 2007. Virulence diversity in the population of Bipolaris sorokiniana. Plant Dis. 91:814-821.

18. Ghazvini, H., and Tekauz, A. 2008. Host-pathogen interactions among barley genotypes and Bipolaris sorokiniana isolates. Plant Dis. 92:225-233.

19. Gurung, S., Goodwin, S. B., Kabbage, M., Bockus, W. W., and Adhikari, T. B. 2011. Genetic differentiation at microsatellite loci among populations of Mycosphaerella graminicola from California, Indiana, Kansas, and North Dakota. Phytopathology 101:1251-1259.

20. Hedrick, P. W. 2005. A standardized genetic differentiation measure. Evolution 59:1633-1638.

21. Ibeagha, A. E., Huckelhoven, R., Schafer, P., Singh, D. P., and Kogel, K.-H. 2005. Model wheat genotypes as tools to uncover effective defense mechanisms against the hemibiotrophic fungus Bipolaris sorokiniana. Phytopathology 95:528-532.

22. Joshi, A. K., Ortiz-Ferrara, G., Crossa, J., Singh, G., Sharma, R. C., Chand, R., and Parsad, R. 2007. Combining superior agronomic performance and terminal heat tolerance with resistance to spot blotch (Bipolaris sorokiniana) of wheat in the warm humid Gangetic Plains of South Asia. Field Crops Res. 103:53-61

23. Knight, N. L., Platz, G. J., Lehmensiek, A., and Sutherland, M. W. 2010. An investigation of genetic variation among Australian isolates of Bipolaris sorokiniana from different cereal tissues and comparison of their abilities to cause spot blotch on barley. Australas. Plant Pathol. 39:207-216.

24. Kumar, U., Joshi, A. K., Kumar, S., Chand, R., and Roder, M. S. 2009. Mapping of resistance to spot blotch caused by Bipolaris sorokiniana in spring wheat. Theor. Appl. Genet. 118:783-793.

25. Kumar, U., Joshi, A. K., Kumar, S., Chand, R., and Roder, M. S. 2010 Quantitative trait loci for resistance to spot blotch caused by Bipolaris soro 
kiniana in wheat (T. aestivum L.) lines 'Ning 8201' and 'Chirya 3'. Mol. Breeding 26:477-491.

26. Ledingham, R. J., Atkinson, T. G., Horricks, J. S., Mills, J. T., Piening, L. J., and Tinline, R. D. 1973. Wheat losses due to common root rot in the prairies of Canada, 1969-71. Can. Plant Dis. Surv. 53:113-122.

27. Limpert, E., and Muller, K. 1994. Designation of pathotypes of plant pathogens. J. Phytopathol. 140:346-358.

28. Liu, Z. H., Zhong, S., Stasko, A. K., Edwards, M. C., and Friesen, T. L. 2012. Virulence profile and genetic structure of a North Dakota population of Pyrenophora teres f. teres, the causal agent of net form net blotch of barley. Phytopathology 102:539-546.

29. Mahto, B. N., Gurung, S., and Adhikari, T. B. 2010. Morphological and pathogenic variability of Cochliobolus sativus from Nepal. (Abstr.) Phytopathology 100:S76.

30. Mahto, B. N. Gurung, S., and Adhikari, T. B. 2011. Assessing genetic resistance to spot blotch, Stagonospora nodorum blotch and tan spot in wheat from Nepal. Eur. J. Plant Pathol. 131:249-260.

31. Meirmans, P. G., and Hedrick, P. W. 2011. Assessing population structure: Fst and related measures. Mol. Ecol. Resour. 11:5-18.

32. Neupane, A. C., Sharma, R. C., Duveiller, E., and Shrestha, S. M. 2010. Sources of Cochliobolus sativus inoculum causing spot blotch under warm wheat growing conditions in South Asia. Cereal Res. Commun. 38:541-549.

33. Neupane, R. B., Sharma, R. C., Duveiller, E., Ortiz-Ferrara, G., Ojha, B. R., Rosyara, U. R., Bhandari, D., and Bhatta, M. R. 2009. Major gene controls of field resistance to spot blotch in wheat genotypes 'Milan/Shanghai \#7' and 'Chirya 3.' Plant Dis. 91:692-697.

34. Okuyama, L. A., Federizzi, L. C., and Neto, J. F. B. 2005. Grain yield stability of wheat genotypes under irrigated and non-irrigated conditions. Brazilian Arch. Biol. Technol. 48:697-704.

35. Pritchard, J. K., Stephens, M., and Donnelly, P. 2000. Inference of population structure using multilocus genotype data. Genetics 155:945-959.

36. Raemaekers, R. H. 1991. Revised Checklist of Plant Diseases in Zambia. Belgian Development Cooperation Project, Chilanga, Zambia.

37. Ruckstuhl, M. 1998. Bipolaris sorokiniana: studies on its epidemiology and population structure in the rice-wheat cropping pattern of Nepal. Pages 88106 in: Helminthosporium Blights of Wheat: Spot Blotch and Tan Spot. E. Duveiller, H. J. Dubin, J. Reeves, and A. McNab, eds. International Maize and Wheat Improvement Center, CIMMYT, El Batan, Mexico, DF.

38. SAS Institute Inc. 2010. SAS OnlineDoc, Version 9.2, SAS Institute, Cary, $\mathrm{NC}$.

39. Shah, D. A., and Madden, L. V. 2004. Nonparametric analysis of ordinal data in designed factorial experiments. Phytopathology 94:33-43.

40. Sharma, R. C., and Duveiller, E. 2007. Advancement toward new spot blotch resistant wheats in South Asia. Crop Sci. 47:961-968.

41. Sharma, R. C., Duveiller, E., Ahmed, F., Arun, B., Bhandari, D., Bhatta, M. R., Chand, R., Chaurasiya, P. C. P., Gharti, D. B., Hossain, M. H., Joshi, A. K., Mahto, B. N., Malaker, P. K., Reza, M. A., Rahman, M., Samad, M. A.,
Shaheed, M. A., Siddique, A. B., Singh, A. K., Singh, K. P., Singh, R. N. and Singh, S. P. 2004. Helminthosporium leaf blight resistance and agronomic performance of wheat genotypes across warm regions of South Asia. Plant Breed. 123:520-524.

42. Sharma, R. C., Duveiller, E., and Ortiz-Ferrara, G. 2007. Progress and challenge towards reducing wheat spot blotch threat in the Eastern Gangetic Plains of South Asia: is climate change already taking its toll? Field Crops Res. 103:109-118.

43. Sharma, R. C., Pandey-Chhetri, B., and Duveiller, E. 2006. Heritability estimates of spot blotch resistance and its association with other traits in spring wheat crosses. Euphytica 147:317-327.

44. Siddique, A. B., Hossain, M. H., Duveiller, E, and Sharma, R. C. 2006. Progress in wheat resistance to spot blotch in Bangladesh. J. Phytopathol. 154:16-22.

45. Svobodova, L. L., Minarikova, V., Kucera, L., and Pereyra, S. A. 2011 Structure of the Cochliobolus sativus population variability. Plant Pathol. Online publication. doi:10.1111/j.1365-3059.2011.02547.x

46. Tamang, P., and Stein, J. 2011. Collection of Cochliobolus sativus isolates and assessment of virulence pattern. (Abstr.) Phytopathology. 101:S2.8.

47. USDA. 2009. Agricultural Statistics. United States Department of Agriculture National Agricultural Statistics Service, United States Government Printing Office, Washington, DC.

48. Valjavec-Gratian, M., and Steffenson, B. J. 1997. Pathotypes of Cochliobolus sativus on barley in North Dakota. Plant Dis. 81:1275-1278.

49. VanderPlank J. E. 1968. Disease Resistance in Plants. Academic, New York (2nd ed. 1984. Academic, Orlando, FL.)

50. Vos, P., Hogers, R., Bleeker, M., Reijans, M., van de Lee, T., Hornes, M., Freijters, A., Pot, J., Peleman, J., Kuiper, M., and Zabeau, M. 1995. AFLP: a new concept for DNA fingerprinting. Nucleic Acids Res. 23:4407-4414.

51. White, T. J., Bruns, T., Lee, S., and Taylor, J. 1990. Amplification and direct sequencing of fungal ribosomal DNA for phylogenetics. Pages 315-322 in: PCR Protocols: A Guide to the Methods and Applications. M. A. Innis, D. H. Gelfand, J. J. Sninsky, and T. J. White, eds. Academic Press, San Diego, CA.

52. Wildermuth, G. B. 1986. Geographic distribution of common root rot and Bipolaris sorokiniana in Queensland wheat soils. Aust. J. Exp. Agric. 26:601-606.

53. Windels, C. E., Lamb, J. A., and Cymbaluk, T. E. 1992. Common root rot and yield responses in spring wheat from chloride application to soil in northwestern Minnesota. Plant Dis. 76:908-911.

54. Yeh, F. C., Yang, R. C., Boyle, T. B. J., Ye, Z. H., and Mao, J. X. 1997. POPGENE, the User Friendly Shareware for Population Genetic Analysis Molecular Biology and Biotechnology Center, University of Alberta, Edmonton, Canada.

55. Zadoks, J. C., Chang, T. T., and Conzak, C.F. 1974. A decimal code for the growth stages of cereals. Weed Res. 14:415-21.

56. Zhong, S., and Steffenson, B. J. 2001. Virulence and molecular diversity of Cochliobolus sativus. Phytopathology 91:469-476. 